\title{
Quantifying wall turbulence via a symmetry approach. Part 2. Reynolds stresses
}

\author{
Xi Chen ${ }^{1,2}$, Fazle Hussain ${ }^{1,2}$ and Zhen-Su She ${ }^{1, \dagger}$ \\ ${ }^{1}$ State Key Laboratory for Turbulence and Complex Systems and Department of Mechanics, \\ College of Engineering, Peking University, Beijing, 100871, China \\ ${ }^{2}$ Department of Mechanical Engineering, Texas Tech University, TX 79409-1021, USA
}

(Received 21 December 2017; revised 5 May 2018; accepted 6 May 2018; first published online 5 July 2018)

We present new scaling expressions, including high-Reynolds-number $(R e)$ predictions, for all Reynolds stress components in the entire flow domain of turbulent channel and pipe flows. In Part 1 (She et al., J. Fluid Mech., vol. 827, 2017, pp. 322-356), based on the dilation symmetry of the mean Navier-Stokes equation a four-layer formula of the Reynolds shear stress length $\ell_{12}$ - and hence also the entire mean velocity profile (MVP) - was obtained. Here, random dilations on the second-order balance equations for all the Reynolds stresses (shear stress $-\overline{u^{\prime} v^{\prime}}$, and normal stresses $\overline{u^{\prime} u^{\prime}}, \overline{v^{\prime} v^{\prime}}, \overline{w^{\prime} w^{\prime}}$ ) are analysed layer by layer, and similar four-layer formulae of the corresponding stress length functions $\ell_{11}, \ell_{22}, \ell_{33}$ (hence the three turbulence intensities) are obtained for turbulent channel and pipe flows. In particular, direct numerical simulation (DNS) data are shown to agree well with the four-layer formulae for $\ell_{12}$ and $\ell_{22}$ - which have the celebrated linear scalings in the logarithmic layer, i.e. $\ell_{12} \approx \kappa y$ and $\ell_{22} \approx \kappa_{22} y$. However, data show an invariant peak location for $\overline{w^{\prime} w^{\prime}}$, which theoretically leads to an anomalous scaling in $\ell_{33}$ in the log layer only, namely $\ell_{33} \propto y^{1-\gamma}$ with $\gamma \approx 0.07$. Furthermore, another mesolayer modification of $\ell_{11}$ yields the experimentally observed location and magnitude of the outer peak of $\overline{u^{\prime} u^{\prime}}$. The resulting $-\overline{u^{\prime} v^{\prime}}, \overline{u^{\prime} u^{\prime}}, \overline{v^{\prime} v^{\prime}}$ and $\overline{w^{\prime} w^{\prime}}$ are all in good agreement with DNS and experimental data in the entire flow domain. Our additional results include: (1) the maximum turbulent production is located at $y^{+} \approx 12$; (2) the location of peak value $-\overline{u^{\prime} v_{p}^{\prime}}$ has a scaling transition from $5.7 R e_{\tau}^{1 / 3}$ to $1.5 R e_{\tau}^{1 / 2}$ at $R e_{\tau} \approx 3000$, with a $1+\overline{u^{\prime} v_{p}^{\prime}}$ scaling transition from $8.5 R e_{\tau}^{-2 / 3}$ to $3.0 R e_{\tau}^{-1 / 2}\left(R e_{\tau}\right.$ the friction Reynolds number); (3) the peak value $\overline{w^{\prime} w_{p}^{\prime}}{ }_{p}^{+} \approx 0.84 R e_{\tau}^{0.14}\left(1-48 / R e_{\tau}\right)$; (4) the outer peak of $\overline{u^{\prime} u^{\prime}}$ emerges above $R e_{\tau} \approx 10^{4}$ with its location scaling as $1.1 R e_{\tau}^{1 / 2}$ and its magnitude scaling as $2.8 R e_{\tau}^{0.09}$; (5) an alternative derivation of the log law of Townsend (1976, The Structure of Turbulent Shear Flow, Cambridge University Press), namely, $\overline{u^{\prime} u^{\prime}} \approx-1.25 \ln y+1.63$ and $\overline{w^{\prime} w^{\prime}} \approx-0.41 \ln y+1.00$ in the bulk.

Key words: pipe flow boundary layer, turbulence theory, turbulent boundary layers 


\section{Introduction}

The mean effect of turbulence is the primary question in turbulent flows, and mean quantities in wall-bounded turbulent flows have been studied for centuries. Yet even for canonical flows, e.g. turbulent channel, pipe and boundary layer flows, predictions of mean profiles continue to remain a challenge (Marusic et al. 2010b; Smits \& Marusic 2013). There have been numerous theoretical attempts to predict the mean velocity scaling (Wosnik, Castillo \& George 2000; Monkewitz, Chauhan \& Nagib 2007; Jones, Nickels \& Marusic 2008; L'vov, Procaccia \& Rudenko 2008; Nagib \& Chauhan 2008; Klewicki 2013; Luchini 2017), but much fewer on the Reynolds stresses (including turbulence intensities $\overline{u^{\prime} u^{\prime}}, \overline{v^{\prime} v^{\prime}}$ and $\overline{w^{\prime} w^{\prime}}$ in streamwise $x$, wall-normal $y$ and spanwise $z$ directions, respectively). For the stresses, an important conceptual model is the wall-attached hypothesis by Townsend (1976). It suggests quantitative descriptions for turbulent eddies (in a statistical sense) and leads to constant profiles for active motions $\left(-\overline{u^{\prime} v^{\prime}}, \overline{v^{\prime} v^{\prime}}\right)$ and $\log$ profiles for inactive motions $\left(\overline{u^{\prime} u^{\prime}}, \overline{w^{\prime} w^{\prime}}\right)$ in the bulk flow (Davidson et al. 2011), i.e.

$$
\begin{gathered}
-\overline{u^{\prime} v^{+}}=B_{12}, \\
\overline{v^{\prime} v^{\prime}}=B_{22}, \\
\overline{u^{\prime} u^{\prime}}=B_{11}-A_{11} \ln y, \\
\overline{w^{\prime} w^{\prime}}=B_{33}-A_{33} \ln y,
\end{gathered}
$$

where $A_{11}, A_{33}, B_{11}, B_{12}, B_{22}$ and $B_{33}$ are all constants, + denotes values in wall units, and $y$ is the wall distance normalized by half-channel height or pipe radius $R$. This hypothesis has received acute attention recently (Marusic \& Kunkel 2003; Davidson \& Krogstad 2009; Meneveau \& Marusic 2013; Vassilicos et al. 2015; Laval et al. 2017), and has been tested and even further developed against more accurate measurements - both DNS and experiment (Morrison, McKeon \& Smits 2004; Hultmark et al. 2012; Rosenberg et al. 2013; Sillero, Jimenez \& Moser 2013; Lee \& Moser 2015; Willert et al. 2017).

Although Perry \& Chong (1982) and Perry, Henbest \& Chong (1986) further developed a derivation of the $\overline{u^{\prime} u^{\prime}} \log$ profile by invoking the $k_{x}^{-1}$ spectrum, such a spectrum was found only at small Reynolds number $\left(R e_{\tau} \leqslant 3300\right)$ in Princeton/ONR Superpipe (Rosenberg et al. 2013); in contrast, the log profile of $\overline{u^{\prime} u^{\prime}}$ is observed only at high Reynolds number $\left(R e_{\tau} \geqslant 20000\right)$ (Hultmark et al. 2012). Also, for channels, Lee \& Moser (2015) found the $k_{x}^{-1}$ spectrum in their simulation $\left(R e_{\tau} \approx 5200\right)$, but no $\log$ profile of $\overline{u^{\prime} u^{\prime}}$. Thus, building the $\log$ profile on the $k_{x}^{-1}$ spectrum is not well founded (Hultmark 2012); we have an alternative explanation for the log profile as discussed later. In addition to the $\log$ profile (restricted in a narrow flow domain), composite formulae for the entire $\overline{u^{\prime} u^{\prime}}$ profile were proposed by Marusic \& Kunkel (2003) and Smits (2010) utilizing the wall-attached eddy concept. Panton (2007) postulated a $\overline{u^{\prime} u^{\prime}}$ model for the entire channel flow using the composite asymptotic expansions, and Alfredsson, Segalini \& Orlu (2011), Alfredsson, Orlu \& Segalini (2012) developed an inner-outer model for $\overline{u^{\prime} u^{\prime}}$ in a turbulent boundary layer (TBL) based on the mean velocity scaling. All the above-mentioned composite formulae involve many free parameters and are difficult to extend to other stress components or other flows (Smits 2010).

The $R e$-scaling of $\overline{u^{\prime} u^{\prime}}$ peaks (location and magnitude) is also controversial (Örlü \& Alfredsson 2013): for the inner peak, while the Princeton pipe shows an invariant magnitude for $R e_{\tau}$ above 3300 (Hultmark et al. 2012), recent pipe data in the 
CICLoPE facility (Willert et al. 2017) show an increasing magnitude with increasing $R e$ up to $R e_{\tau} \approx 40000$. It is unclear whether this growth is solely due to a finite $R e$ effect, or it suggests that the viscous scaling is invalid for fluctuations (Degraaff \& Eaton 2000; Buschmann, Indinger \& Gadelhak 2009) since the inner-outer interactions are very effective (Hutchins \& Marusic 2007b; Marusic, Mathis \& Hutchins 2010a; McKeon 2017). For the outer peak, McKeon \& Sharma (2010) and Moarref et al. (2013) developed a 'critical layer' framework to understand its $y$-location scaling in pipes (argued to scale as $\operatorname{Re}_{\tau}^{2 / 3}$ ). Moreover, there are efforts devoted to identify the characteristic flow structures (Jimenez 2012), e.g. the large scale motions (LSM) (Adrian 2007) and very large scale motions (VLSM) (Hutchins \& Marusic 2007a,b), which are suggested to lead to the emergence of the outer peak (Hultmark et al. 2013; Vincenti et al. 2013; Vallikivi, Ganapathisubramani \& Smits 2015).

While considerable efforts have been devoted to studies of $\overline{u^{\prime} u^{\prime}}$, much less attention has been given to other Reynolds stress components; the study of these components is essential to unveil how fluctuations in different directions are coupled. In She, Chen \& Hussain (2017) (hereafter cited as Part 1), we obtained a four-layer (consisting of viscous sublayer, buffer layer, bulk layer and core layer) formula for the Reynolds shear stress length $\ell_{12}$ (defined later) - and hence also the entire mean velocity profile (MVP) - by employing the dilation symmetry of the mean Navier-Stokes equation. Here, random dilations on the second-order balance equations for all the Reynolds stresses (shear stress $-\overline{u^{\prime} v^{\prime}}$, and normal stresses $\overline{u^{\prime} u^{\prime}}, \overline{v^{\prime} v^{\prime}}, \overline{w^{\prime} w^{\prime}}$ ) are analysed layer by layer. Similar four-layer formulae of the corresponding stress length functions $\ell_{11}, \ell_{22}$, $\ell_{33}$ are obtained for turbulent channel and pipe flows (exactly parallel flows), leading to analytical descriptions of all three Reynolds normal stresses (or intensities) in the entire flow domain.

In particular, DNS data agree well with the four-layer formulae for $\ell_{12}$ and $\ell_{22}$, while anomalous scaling modifications are needed for $\ell_{11}$ and $\ell_{33}$ in the log layer. The resulting $-\overline{u^{\prime} v^{\prime}}, \overline{u^{\prime} u^{\prime}}, \overline{v^{\prime} v^{\prime}}$ and $\overline{w^{\prime} w^{\prime}}$ are all in good agreement with DNS as well as experimental data in the entire flow domain. Furthermore, the aforementioned $y$ scaling as well as the Re-scaling of the peak values and locations of all the stress components are formulated in terms of the multilayer parameters (layer thicknesses and scaling exponents), enabling new predictions. For example, as we show below, the peak location of $-\overline{u^{\prime} v^{\prime}}$ can be given as a function of the von Kármán constant $\kappa$ (note the recently reported $\kappa=0.446 \pm 0.008$ in CICLoPE experiments by Nagib et al. (2017) - quite consistent with our $\kappa \approx 0.45$ in Part 1 ) and the buffer layer thickness $y_{b u f}^{+}$, and it has a scaling transition from $\operatorname{Re}_{\tau}^{1 / 3}$ to $\operatorname{Re}_{\tau}^{1 / 2}$ at a critical $\operatorname{Re}_{\tau}^{c} \approx$ 3000. The $R e_{\tau}^{1 / 2}$ scaling was also found in Sreenivasan (1988); however, the $R e_{\tau}^{1 / 3}$ scaling for small $R e$ is new - agreeing well with DNS data. Furthermore, previously argued $\log$ profiles of inactive motions are obtained as local flow approximations, with a theoretical determination of its coefficients: $A_{11}=1.25, B_{11}=1.63$, and $A_{33}=0.41$, $B_{33}=1.00$; these values are very close to the empirical values of $A_{11}=1.25, B_{11}=1.61$ by Hultmark et al. (2012), and of $A_{33}=0.387$ and $B_{33}=1.08$ by Lee \& Moser (2015). Moreover, predictions of all Reynolds stress profiles at $R e$ one decade larger than current available data - i.e. $-\overline{u^{\prime} v^{\prime}}, \overline{v^{\prime} v^{\prime}}$ and $\overline{w^{\prime} w^{\prime}}$ at $R e_{\tau}=50000$, and $\overline{u^{\prime} u^{\prime}}$ at $R e_{\tau}=10^{6}$ - are also presented in this paper, awaiting future measurements for validation. Note that the current work characterizes the physically sound multilayer structure for all the Reynolds stresses, smoothly varying in space, needing no spectral description, and thus involving far fewer parameters than the previous models.

The paper is organized as follows. Section 2 presents the Lie-group analysis for the second-order balance equations of channel and pipe flows, which extends the analysis 
reported in Part 1 . In $\S \S 3$ and 4 , we validate the four-layer predictions for $-\overline{u^{\prime} v^{\prime}}$ and $\overline{v^{\prime} v^{\prime}}$, respectively. Results of $\overline{w^{\prime} w^{\prime}}$ with an additional anomalous scaling modification are presented in $\S 5$. Section 6 discusses $\overline{u^{\prime} u^{\prime}}$. Section 7 contains a summary with future perspectives.

\section{Random dilation for the balance equations}

This section is devoted to a complete treatment of the mean momentum equation and the second-order balance equations for the four Reynolds stresses under a random dilation transformation. The goal is to discover dilation groups with enough parameters which leave balance equations invariant in different regions of $y$, and then to define the multilayer structure for the length functions. The basic idea is that the length functions represent the spatial scales of (statistical) turbulent eddies, whose variations in each of the layers are assumed to be self-similar viz. satisfying dilation invariance. We further ask to what extent the dilation invariance of these lengths can be related to the symmetry of the balance equations, as the symmetry enables a general expression for dilation invariants. Then, noting that the power law is just a specific expression of dilation invariance, two more expressions (ansatze) are postulated in Part 1 - one describing the defect power law in the bulk flow region (i.e. dilation symmetry broken in the length but retained in its derivative), and the other describing a generalized invariance across two adjacent layers. These ansatze are also validated here, leading to composite formulae for the length functions and quantitative descriptions of Reynolds stresses in the entire flow domain.

Taking channel flow for example, the ensemble-averaged mean momentum and Reynolds stress equations are

$$
\begin{aligned}
& \frac{\partial^{2} U^{+}}{\partial y^{+2}}-\frac{\partial \overline{u^{\prime} v^{\prime}}}{\partial y^{+}}=-\frac{1}{R e_{\tau}}, \\
& -{\overline{u^{\prime} v^{\prime}}}^{+} \frac{\partial U^{+}}{\partial y^{+}}-\overline{u^{\prime} \frac{\partial p^{\prime}}{\partial x}}-\frac{\partial}{\partial y^{+}}\left(\frac{1}{2} \overline{u^{\prime} u^{\prime} v^{\prime}}\right)-\overline{\left|\nabla u^{\prime}\right|^{+}}+\frac{\partial^{2}}{\partial y^{+2}}\left(\frac{1}{2}{\overline{u^{\prime 2}}}^{+}\right)=0, \\
& {\overline{-v^{\prime} \frac{\partial p^{\prime}}{\partial y}}}^{+}-\frac{\partial}{\partial y^{+}}\left(\frac{1}{2}{\overline{v^{\prime} v^{\prime} v^{\prime}}}^{+}\right)-{\overline{\left|\nabla v^{\prime}\right|^{+}}}^{2}+\frac{\partial^{2}}{\partial y^{+2}}\left(\frac{1}{2}{\overline{v^{\prime 2}}}^{+}\right)=0, \\
& {\overline{w^{\prime}}}_{\frac{\partial p^{\prime}}{\partial z}}^{+}-\frac{\partial}{\partial y^{+}}\left(\frac{1}{2}{\overline{w^{\prime} w^{\prime} v^{\prime}}}^{+}\right)-{\overline{\left|\nabla w^{\prime}\right|^{+}}}^{2}+\frac{\partial^{2}}{\partial y^{+2}}\left(\frac{1}{2}{\overline{w^{\prime 2}}}^{+}\right)=0, \\
& -\frac{1}{2}{\overline{v^{\prime} v^{\prime}}}^{+} \frac{\partial U^{+}}{\partial y^{+}}-\frac{1}{2} \overline{u^{\prime} \frac{\partial p^{\prime}}{\partial y}}+\frac{1}{2} \overline{v^{\prime} \frac{\partial p^{\prime}}{\partial x}}-\frac{\partial}{\partial y^{+}}\left(\frac{1}{2} \overline{u^{\prime} v^{\prime} v^{\prime}}+\right) \\
& -\overline{\left|\nabla u^{\prime} \cdot \nabla v^{\prime}\right|^{+}}+\frac{\partial^{2}}{\partial y^{+2}}\left(\frac{1}{2} \overline{u^{\prime} v^{\prime}}+\right)=0 \text {. }
\end{aligned}
$$

Here, $\operatorname{Re}_{\tau} \equiv u_{\tau} \delta / v$ is the friction Reynolds number $\left(u_{\tau}\right.$ is the friction velocity and $\delta$ is the pipe radius or half-channel height), the overbar indicates space ( $x$ and $z$ ) and time $(t)$ average, and superscript ' indicates fluctuation.

In Part 1, a multilayer structure for stress length $\ell_{12}$ (see below) is proposed to accurately quantify the mean velocity profile (MVP), which involves three layer thicknesses measured from DNS data of MVP as $y_{s u b}^{+}=9.7$ (for the sublayer), $y_{b u f}^{+}=41$ (for the buffer layer) and $r_{\text {core }}=0.27$ (for the central core layer normalized 
(a)

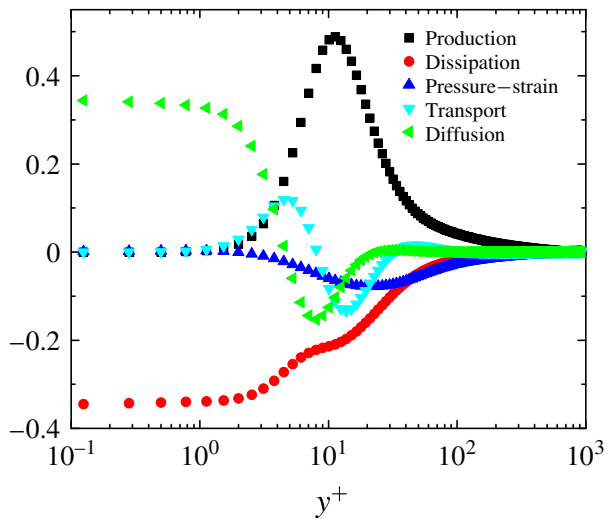

(b)

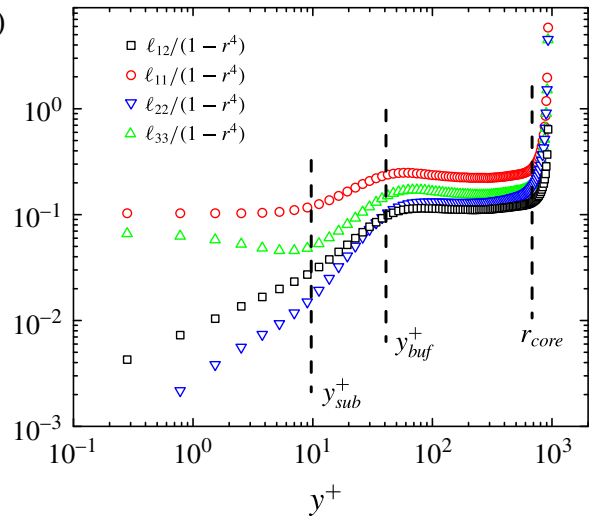

FIgURE 1. (Colour online) (a) Budget of $\overline{u^{\prime} u^{\prime}}(2.1 b)$. (b) Compensated plot of $\ell_{11}, \ell_{12}$, $\ell_{22}$ and $\ell_{33}$ (normalized by the half-channel height) divided by $1-r^{4}$ (Part 1 ). Note that the bulk layer (between $y_{b u f}^{+}$and $r_{\text {core }}$ ) has nearly constant values. Thicknesses of viscous sublayer $\left(y_{\text {sub }}^{+}=9.7\right)$, buffer layer $\left(y_{\text {buf }}^{+}=41\right)$ and core layer $\left(r_{\text {core }}=0.27\right)$ shown by dashed lines. Data from DNS channel flow at $R e_{\tau}=940$ (Hoyas \& Jimenez 2006).

with $\delta$ for channels and pipes). In Part 1, we also showed that they quantify the transitions of balances between different mechanisms (captured in the turbulent kinetic energy equation) in different layers, and symmetry analysis of budget equations in different layers are performed here. Figure 1(a) shows the wall-normal variation of various terms in $(2.1 b)$ for $\overline{u^{\prime} u^{\prime}}$, where the dominant balancing mechanism changes as $y^{+}$increases. In particular, there is a shift from a diffusion-dissipation balance close to the wall to a production-dissipation balance for $y^{+} \gtrsim y_{\text {sub }}^{+}$. Further away from the wall, the pressure-strain term becomes more important and takes over dissipation at $y^{+} \approx y_{b u f}^{+}$, representing significant redistribution of the kinetic energy from the streamwise to the normal and spanwise directions. For $y^{+} \gtrsim y_{b u f}^{+}$, the net production (subtracting the mean shear production by the pressure-strain term) then balances the dissipation, where turbulent transport is negligible. However, near the centre $r \lesssim r_{\text {core }}(r=1-y$ being the distance from the centreline normalized by the half-channel height), turbulent transport takes over production to balance dissipation and pressure-strain - which defines a core layer. Such shifts in the balancing mechanism give rise to the aforementioned four-layer structure. (Note that the current four layers are different from those defined by Wei et al. (2005) and Klewicki et al. (2012) from the balance of the mean momentum equation). Here, the multilayer structure is also visible by the compensated plot (divided by $1-r^{4}$ ) of the lengths (Part 1), as shown in figure 1(b). Below, we present a detailed symmetry analysis for all balance equations like we did for the mean momentum equation in Part 1, so as to develop an analytical framework enabling quantification of the Reynolds stress distributions.

Note a common difficulty in the classical Lie-group analysis: namely, the lack of free group parameters to predict (or explain) scaling laws. In order to circumvent this difficulty, physical considerations are typically invoked to add to the parameters. This has been practiced in the derivation of the Blasius solution for the laminar boundary layer (Cantwell 2002) and similarity solutions for sink-flow boundary layer (Chen \& Hussain 2017), where an inhomogeneous dilation is introduced under the boundary layer approximation. Below, to enlarge the group parameter space, a three-step 
symmetry analysis is developed: first, we carry out a statistical symmetry analysis by employing random dilations. Second, we define a locally valid dilation group by neglecting unimportant terms in the balance equations. Third, we postulate the existence of a set of key similarity variables (hence order function) whose invariants are locally constants (hence having power laws), or satisfy simple invariant ansatze. The lengths introduced in $(2.3 a, b)$ are such similarity variables. Note that their power laws can be validated by empirical data via an a priori test (i.e. without symmetry analysis), as shown in figure 1(b); the symmetry analysis below establishes the connections between the local power laws and the balance mechanisms represented by the budget equations. The specified length functions then lead to the Reynolds stress distributions.

Specifically, the random dilation transformation is introduced as follows:

$$
x_{i}^{*}=q_{i} x_{i}, \quad U^{*}=\lambda_{U} U, \quad R e_{\tau}^{*}=\lambda_{R} R e_{\tau}, \quad u_{i}^{\prime *}=\lambda_{i} u_{i}^{\prime}, \quad p^{*}=\lambda_{p} p^{\prime},
$$

where superscript $*$ indicates transformed variables (all variables are normalized by wall units and the superscript + is omitted); $q_{i}, \lambda_{U}$ and $\lambda_{R}$ are non-random, while $\lambda_{i}$ and $\lambda_{p}$ are random variables with zero mean. Note that (2.2) is reasonable since turbulent fluctuations are random. The dilation correlation coefficients of $\left\{\lambda_{i}\right\}$ such as $\overline{\lambda_{i} \ldots \lambda_{k}}$ - which are independent of correlations between velocities and pressure provide extra group parameters enabling the study of the dilation symmetry of the (infinite) hierarchy of high-order correlation equations (Oberlack 2001; Marati et al. 2006; Oberlack \& Rosteck 2010; Frewer \& Khujadze 2016). For example, for the second-order correlations, $\overline{\lambda_{1} \lambda_{2}}$ differs from $\overline{\lambda_{1} \lambda_{1}}$ and $\overline{\lambda_{2} \lambda_{2}}$; note that in situations when $\overline{\lambda_{1} \lambda_{1}}$ and $\overline{\lambda_{2} \lambda_{2}}$ are equal (see later), $\overline{\lambda_{1} \lambda_{2}}$ can still be different. This allows different dilations on the Reynolds normal and shear stresses, as shown later.

In this paper, four lengths are defined by the mean shear $S^{+}=\partial U^{+} / \partial y^{+}$and the Reynolds stresses $W_{i j}^{+}=\left|\overline{u_{i}^{\prime} u_{j}^{\prime}}\right|^{+}$(where $\|$denotes the absolute value, particularly for $\overline{u_{1}^{\prime} u_{2}^{\prime}}$ or $\overline{u^{\prime} v^{\prime}}$ since it is negative in shear flows):

$$
\begin{array}{cc}
\ell_{12}^{+}=W_{12}^{+1 / 2} / S^{+}, & \ell_{i i}^{+}=W_{i i}^{+1 / 2} / S^{+}, \\
W_{12}^{+}=\left(\ell_{12}^{+} S^{+}\right)^{2}, & W_{i i}^{+}=\left(\ell_{i i}^{+} S^{+}\right)^{2},
\end{array}
$$

where $i=1,2,3$ (no summation for indices unless otherwise stated). Under the dilations (2.2), the stresses and the lengths are dilated as

$$
W_{i j}^{+*}=\overline{\lambda_{i} \lambda_{j}} W_{i j}^{+}, \quad \ell_{i j}^{+*}=\left(\sqrt{\overline{\lambda_{i} \lambda_{j}}} q_{2} / \lambda_{U}\right) \ell_{i j}^{+} .
$$

Note that these lengths, forming a family of multiple components, characterize different sets of eddies contributing to anisotropic transport of the Reynolds stress tensor. They are considered here as similarity variables satisfying three dilation invariant ansatze, similar to $\ell_{12}$ in Part 1 . The local dilation invariance actually indicates that turbulent eddies are self-similar; namely, within each layer, eddies' characteristic scales at different $y$-locations are related by a simple power law. The previous linear $y$-scaling of the wall-attached eddy size in the log layer (Alamo et al. 2006; Jimenez 2012) is only one of the layers, and the linear scaling can be derived by the dilation symmetry analysis as below. Across different layers, eddies' scales obey a generalized power law, following the third ansatz postulated in Part 1, so that we establish a multilayer similarity of the eddies across the entire flow domain. 
Below, we apply (2.2) to $(2.1 a-e)$ to obtain concrete expressions for the lengths in $(2.3 e, f)$. Particularly, the linear scaling in the log layer is obtained by equating the dilation coefficients of all terms in (2.1). In the viscous sublayer, and bulk and core layers, we remove some unimportant terms so as to reduce the symmetry constraints, and hence to increase the freedom of new scaling. In the buffer layer, two large terms (i.e. dissipation and diffusion) are of opposite sign and hence combined so as to capture the higher-order term in $(2.1 b)$. This way, different directional dilations are obtained in the viscous sublayer, and buffer, bulk and core layers, where the balance mechanism in each layer is different. The local power-law exponents for all the lengths can be determined using expansion or other theoretical arguments as in Part 1, and a generalized Lie-group ansatz (i.e. the third ansatz in Part 1) connects power laws of adjacent layers together, so as to yield the four-layer composite profile for each length in the entire flow domain.

\subsection{Homogeneous dilations in the log layer}

Substituting (2.2) into (2.1a)-(2.1e) and requiring the same proportional coefficients for all the terms in the equations (needed to obtain invariant transformed equations) yield the following relations for the correlations of random dilation variables $\left\{\lambda_{i}\right\}$ :

$$
\begin{aligned}
& \lambda_{U} / q_{2}^{2}=\overline{\lambda_{1} \lambda_{2}} / q_{2}=1 / \lambda_{R}, \\
& \overline{\lambda_{1} \lambda_{2}} \lambda_{U} / q_{2}=\overline{\lambda_{1} \lambda_{p}} / q_{1}=\overline{\lambda_{1} \lambda_{1} \lambda_{2}} / q_{2}=\overline{\lambda_{1} \lambda_{1}} / q_{1}^{2}=\overline{\lambda_{1} \lambda_{1}} / q_{2}^{2}=\overline{\lambda_{1} \lambda_{1}} / q_{3}^{2}, \\
& \overline{\lambda_{2} \lambda_{p}} / q_{2}=\overline{\lambda_{2} \lambda_{2} \lambda_{2}} / q_{2}=\overline{\lambda_{2} \lambda_{2}} / q_{1}^{2}=\overline{\lambda_{2} \lambda_{2}} / q_{2}^{2}=\overline{\lambda_{2} \lambda_{2}} / q_{3}^{2}, \\
& \overline{\lambda_{3} \lambda_{p}} / q_{3}=\overline{\lambda_{3} \lambda_{3} \lambda_{2}} / q_{2}=\overline{\lambda_{3} \lambda_{3}} / q_{1}^{2}=\overline{\lambda_{3} \lambda_{3}} / q_{2}^{2}=\overline{\lambda_{3} \lambda_{3}} / q_{3}^{2}, \\
& \overline{\lambda_{2} \lambda_{2}} \lambda_{U} / q_{2}=\overline{\lambda_{1} \lambda_{p}} / q_{2}=\overline{\lambda_{2} \lambda_{p}} / q_{1}=\overline{\lambda_{1} \lambda_{2} \lambda_{2}} / q_{2}=\overline{\lambda_{1} \lambda_{2}} / q_{1}^{2}=\overline{\lambda_{1} \lambda_{2}} / q_{2}^{2}=\overline{\lambda_{1} \lambda_{2}} / q_{3}^{2} \text {. }
\end{aligned}
$$

These necessarily require homogeneous dilations in the three directions, i.e. $q_{1}=q_{2}=$ $q_{3}=q$, and also

$$
\begin{gathered}
\lambda_{U}=1 / q, \quad \lambda_{R}=q^{3}, \\
\overline{\lambda_{1} \lambda_{1}}=\overline{\lambda_{2} \lambda_{2}}=\overline{\lambda_{1} \lambda_{2}}=1 / q^{2}, \\
\overline{\lambda_{1} \lambda_{p}}=\overline{\lambda_{2} \lambda_{p}}=\overline{\lambda_{1} \lambda_{2} \lambda_{2}}=\overline{\lambda_{1} \lambda_{1} \lambda_{2}}=\overline{\lambda_{2} \lambda_{2} \lambda_{2}}=1 / q^{3} .
\end{gathered}
$$

Accordingly, the Reynolds stresses $W_{i j}^{+}$are dilated by a factor of $\overline{\lambda_{i} \lambda_{j}}=1 / q^{2}$ (for $i, j$, = $1,2)$, and the mean shear $S$ by a factor of $\lambda_{U} / q=1 / q^{2}$, so that the length functions, according to definitions in $(2.3 a, b)$, are dilated as

$$
\ell_{i j}^{+*}=\sqrt{\overline{\lambda_{i} \lambda_{j}}} /\left(\lambda_{U} / q\right) \ell_{i j}^{+}=q \ell_{i j}^{+} .
$$

In this case, the dilation invariants consisting of $y^{+}$and $\ell_{i j}^{+}$are

$$
I_{i j}^{\log }=\ell_{i j}^{+} / y^{+} .
$$

Following Part 1 , we assume constant dilation invariants (i.e. $I_{i j}=$ const.) to obtain possible (or candidate) solutions of the balance equations, i.e.

$$
\ell_{i j}^{+}=I_{i j}^{l o g} y^{+}, \quad I_{i j}^{l o g}=\text { const. }
$$


This is the standard (canonical) case where no direction is preferred, and all the lengths $\ell_{i j}$ scale as the wall distance $(y)$ - also a simple consequence of dimensional analysis.

In particular, the well-known $W_{12}^{+} \approx 1$ gives rise to $S^{+} \approx 1 /\left(I_{12}^{\text {log }} y^{+}\right)$and hence the mean velocity $U^{+} \approx\left(1 / I_{12}^{l o g}\right) \log y^{+}+B$; this shows that the Kármán constant is just one of our dilation invariants: $I_{12}^{\text {log }}$. Note that the dilation invariance of $W_{12}^{+}$actually is broken since $W_{12}^{+} \approx 1$ against $W_{12}^{+*}=W_{12}^{+} / q^{2}$ (unless $q=1$, which would indicate no dilation), but the length function $\ell_{12}$ still holds its dilation invariance: $\ell_{12}^{+*}=q \ell_{12}^{+}$(this is why $\ell_{i j}$, not $W_{i j}$, is chosen as the similarity variable). This is also found in other layers that we study below.

More interestingly, from (2.8) and $(2.3 c, d), W_{i i}^{+}=W_{12}^{+}\left(I_{i i}^{l o g} / I_{12}^{l o g}\right)^{2}$, indicating that all the Reynolds normal stresses are constants in the log layer (since $W_{12}^{+} \approx 1$ ). These constants are parts of the so-called 'inertial-range' description, widely used in engineering models (Wilcox 2006). The present analysis shows that they arise from a dilation symmetry homogeneous in all directions (when all terms in the balance equations are under the same dilation). Note that the constancy of $W_{11}^{+}$and $W_{33}^{+}$ are against Townsend's log law of inactive motions in (1.3) and (1.4), where more discussions on this issue will be presented later.

Also note that the above analysis shows that dilations of $W_{11}, W_{22}$ and $W_{12}$ are linked, because of the production and pressure-strain terms in the corresponding equations. In contrast, dilation on $W_{33}$ seems to be independent of those on $W_{11}$ or $W_{22}$ (hence is free), since no term in the spanwise kinetic energy equation is coupled with other equations. Here, invoking the isotropic turbulence assumption, $\ell_{33}$ satisfies the same dilation as $\ell_{11}$ or $\ell_{22}$; this argument also applies to the core layer. However, near the wall, the isotropic condition is invalid, and dilations on these lengths should be different. In fact, the homogeneous dilation symmetry is partially broken near the wall since some terms in the balance equations are unimportant. We explore such possibilities below. The analysis will first treat $\ell_{i j}$ (for $i, j=1,2$ ) and then focus on $\ell_{33}$.

\subsection{Directional dilations in the viscous sublayer}

Near the wall, compared to $\partial_{y}^{2}$, the $\partial_{x}^{2}$ and $\partial_{z}^{2}$ terms can be ignored (i.e. the boundary layer approximation). This enables us to drop the $\overline{\lambda_{i} \lambda_{j}} / q_{1}^{2}$ and $\overline{\lambda_{i} \lambda_{j}} / q_{3}^{2}$ terms in (2.4), and hence all $q_{i}$ are not needed to be equal. In this case, let us choose $\lambda_{U}$ and $q_{2}$ as two independent dilation parameters, then all the other dilation parameters are determined from the remnant of (2.4):

$$
\begin{aligned}
& \lambda_{R}=q_{2}^{2} / \lambda_{U}, \quad \overline{\lambda_{1} \lambda_{2}}=\lambda_{U} / q_{2}, \quad \overline{\lambda_{1} \lambda_{1}}=\lambda_{U}^{2}, \quad \overline{\lambda_{2} \lambda_{2}}=1 / q_{2}^{2}, \quad q_{1}=1 / \lambda_{U} . \\
& \overline{\lambda_{1} \lambda_{p}}=\overline{\lambda_{1} \lambda_{2} \lambda_{2}}=\lambda_{U} / q_{2}^{2}, \quad \overline{\lambda_{2} \lambda_{p}}=\overline{\lambda_{2} \lambda_{2} \lambda_{2}}=1 / q_{2}^{3}, \quad \overline{\lambda_{1} \lambda_{1} \lambda_{2}}=\lambda_{U}^{2} / q_{2} .
\end{aligned}
$$

Therefore, the lengths are dilated as

$$
\ell_{11}^{+*}=q_{2} \ell_{11}^{+}, \quad \ell_{12}^{+*}=\sqrt{q_{2} / \lambda_{U}} \ell_{12}^{+}, \quad \ell_{22}^{+*}=\left(1 / \lambda_{U}\right) \ell_{22}^{+} .
$$

Since $\ell_{12}^{+*}=q_{2}^{3 / 2} \ell_{12}^{+}$was obtained in the viscous sublayer due to the scaling $\ell_{12}^{+} \propto y^{+3 / 2}$ (Part 1), we thus have $\lambda_{U}=q_{2}^{-2}$ from the second equation of (2.10), and hence $\ell_{22}^{+*}=$ $q_{2}^{2} \ell_{22}^{+}$. Then, the corresponding dilation invariants are

$$
I_{11}^{s u b}=\ell_{11}^{+} / y^{+}, \quad I_{12}^{s u b}=\ell_{12}^{+} / y^{+3 / 2}, \quad I_{22}^{s u b}=\ell_{22}^{+} / y^{+2} .
$$


Following the first invariant ansatz (i.e. assuming constant invariants) in Part 1, local power laws in the viscous sublayer are obtained as

$$
\ell_{11}^{+}=I_{11}^{s u b} y^{+}, \quad \ell_{12}^{+}=I_{12}^{s u b} y^{+3 / 2}, \quad \ell_{22}^{+}=I_{22}^{s u b} y^{+2} .
$$

This shows that by random dilation of approximate balance equations, we can obtain different power laws for the length functions, in contrast to the uniform linear scaling (2.8) for all the lengths in log layer.

\subsection{Directional dilations in the buffer layer}

The dominant balancing mechanisms in the buffer layer are different from those in the viscous sublayer. While $(2.1 a),(2.1 c)$ and $(2.1 d)$ remain the same as in the viscous sublayer (i.e. ignoring $\partial_{x}^{2}$ and $\partial_{z}^{2}$ in dissipations), both (2.1e) and (2.1b) are different. For the Reynolds shear stress equation (2.1e), diffusion and dissipation are very small (Pope 2000), and hence ignored. For the streamwise stress equation (2.1b), a special consideration is given below.

Recall that in Part 1, we defined an eddy shear length $\ell_{v}^{+}$:

$$
\ell_{v}^{+}=\left(W_{12}^{+} / S^{+}\right)^{3 / 4} / \epsilon_{v}^{1 / 4}
$$

where $\epsilon_{v}=\left(\overline{\left|\nabla u^{\prime}\right|^{+}}{ }^{2}-\partial^{2} \overline{u^{\prime} u^{\prime}} / \partial y^{+2}\right)$ is the net viscous effect (a sum of viscous diffusion and dissipation) and $\nu_{T}^{+}=W_{12}^{+} / S^{+}$is the eddy viscosity. In the viscous sublayer, $W_{12}^{+} / S^{+} \propto y^{+3}$ and $\epsilon_{v} \propto y^{+}$(since the leading order expansions of ${\overline{\left|\nabla u^{\prime}\right|^{+}}}^{2}$ and $\partial^{2}\left(\overline{u^{\prime} u^{\prime}}\right) / \partial y^{+2}$ - which are non-zero values - exactly balance each other (see figure $1 a$ ), leaving the first expansion term as a order of $\left.y^{+}\right)$. Thus, the expansion for the eddy shear length is $\ell_{\nu}^{+} \approx c_{\nu} y^{+2}+$ h.o.t. (where h.o.t. denotes higher-order terms.) Here, using the same buffer layer approximation $\ell_{v}^{+} \approx c_{v} y^{+2}$ as in Part 1 , the net viscous effect is approximated as

$$
\epsilon_{\nu}=\frac{W_{12}^{+3}}{S^{+3} \ell_{\nu}^{+4}}=\frac{W_{12}^{+3}}{S^{+3}\left(c_{\nu} y^{+2}+\text { h.o.t. }\right)^{4}} \approx \frac{W_{12}^{+3}}{S^{+3}\left(c_{\nu} y^{+2}\right)^{4}} .
$$

Substituting (2.14) into $(2.1 b)$ yields

$$
0 \approx S^{+} W_{12}^{+}-\overline{u^{\prime} \partial_{x} p^{\prime}}+\partial_{y^{+}}\left(\frac{1}{2} \overline{u^{\prime} u^{\prime} v^{\prime}}\right)-\frac{W_{12}^{+3}}{S^{+3}\left(c_{\nu} y^{+2}\right)^{4}},
$$

which will replace $(2.1 b)$ to be the approximate streamwise stress equation for the dilation analysis (explained later).

Applying (2.2) to the newly obtained approximated equations yields the following constraints on the correlations of dilation parameters:

$$
\begin{gathered}
\lambda_{R}=q_{2}^{2} / \lambda_{U}, \quad \overline{\lambda_{1} \lambda_{2}}=\lambda_{U} / q_{2}, \quad \lambda_{U}=1 / q_{2}^{3}, \quad q_{1}=q_{2}^{2} \overline{\lambda_{2} \lambda_{2}} / \lambda_{U} \\
\overline{\lambda_{1} \lambda_{p}}=\overline{\lambda_{1} \lambda_{2} \lambda_{2}}=\lambda_{U} \overline{\lambda_{2} \lambda_{2}}, \quad \overline{\lambda_{2} \lambda_{p}}=\overline{\lambda_{2} \lambda_{2} \lambda_{2}}=q_{2}\left(\overline{\lambda_{2} \lambda_{2}}\right)^{2}, \quad \overline{\lambda_{1} \lambda_{1} \lambda_{2}}=\lambda_{U}^{2} / q_{2} .
\end{gathered}
$$

Therefore, the lengths are dilated as

$$
\ell_{11}^{+*}=q_{11} \ell_{11}^{+}, \quad \ell_{12}^{+*}=q_{2}^{2} \ell_{12}^{+}, \quad \ell_{22}^{+*}=q_{22} \ell_{22}^{+},
$$


where $q_{11}=q_{2} \sqrt{\overline{\lambda_{1} \lambda_{1}}} / \lambda_{U}$ and $q_{22}=q_{2} \sqrt{\lambda_{2} \lambda_{2}} / \lambda_{U}$ are two free parameters, since $\overline{\lambda_{1} \lambda_{1}}$ and $\overline{\lambda_{2} \lambda_{2}}$ are free.

Under the constant dilation ansatz, equation (2.17) further leads to

$$
\ell_{11}^{+}=I_{11}^{\text {buf }} y^{+\beta_{11}}, \quad \ell_{12}^{+}=I_{12}^{\text {buf }} y^{+2}, \quad \ell_{22}^{+}=I_{22}^{\text {buf }} y^{+\beta_{22}},
$$

where $q_{11}=q_{2}^{\beta_{11}}$ and $q_{22}=q_{2}^{\beta_{22}}$ are substituted in. Note that $\ell_{12}^{+}=I_{12}^{\text {buf }} y^{+2}$ in the second equation of (2.18) has been obtained in Part 1. Further arguments are invoked to determine the values of $\beta_{11}$ and $\beta_{22}$. Consider the following ratios:

$$
\ell_{11}^{+} / \ell_{12}^{+}=\left(I_{11}^{\text {buf }} / I_{12}^{\text {buf }}\right) y^{+\left(\beta_{11}-2\right)}, \quad \ell_{22}^{+} / \ell_{12}^{+}=\left(I_{22}^{\text {buf }} / I_{12}^{\text {buf }}\right) y^{+\left(\beta_{22}-2\right)} .
$$

From (2.12), $\ell_{11}^{+} / \ell_{12}^{+} \propto 1 / y^{+1 / 2}$ and $\ell_{22}^{+} / \ell_{12}^{+} \propto y^{+1 / 2}$ in the viscous sublayer. We assume that the two ratios representing the geometrical feature (e.g. a two-dimensional shape in the $x-y$ plane) of near-wall eddies remain invariant from the sublayer to the buffer layer - so that $\beta_{11}=3 / 2$ and $\beta_{22}=5 / 2$ in (2.19). The mechanism for these invariant scalings is not clear yet, and is to be studied in future.

Note that the free parameter $q_{11}$ originates from the approximation (2.15). Without this approximation, repeating the same analysis would yield $\overline{\lambda_{1} \lambda_{1}}=\lambda_{U}^{2}, q_{11}=q_{2}$, and hence $\ell_{11}^{+} \propto y^{+}-$the same scaling as in the viscous sublayer (2.12). However, this contradicts the plot in figure $1(b)$, where $\ell_{11}^{+}$shows a distinct scaling transition from the viscous sublayer to the buffer layer. Thus, the approximation (2.14) (hence (2.15)) is necessary.

\subsection{Homogeneous dilations in the core layer}

Similar to Part 1, we change the independent variable from the wall distance $y^{+}$to the distance from the centreline $r=1-y^{+} / R e_{\tau}$, with the corresponding outer scales in the other two directions, namely, $\tilde{x}=x^{+} / R e_{\tau}$ and $\tilde{z}=z^{+} / R e_{\tau}$. Also, we introduce a velocity defect $U_{d}^{+}=U_{c}^{+}-U^{+}$(subscript $c$ indicates centreline value) to replace the mean velocity in the balance equations, so that the centreline condition $U_{d}^{+}=0-$ in analogy to $U^{+}=0$ at the wall - remains unchanged under dilation. Note that if one applies (2.2) (also changing $U^{+}$to $U_{d}^{+}, x^{+}$to $\tilde{x}, y^{+}$to $r$ and $z^{+}$to $\tilde{z}$ ) to the balance equations scaled in outer units, one would obtain uniform dilations $\overline{\lambda_{1} \lambda_{2}}=\overline{\lambda_{i} \lambda_{i}}=q_{i}$ and hence the same power law for all the lengths. However, this contradicts the centre asymptotes:

$$
\ell_{12} \propto 1 / \sqrt{r} ; \quad \ell_{i i} \propto 1 / r
$$

$(i=1,2,3)$, explained as follows. In the limit $r \rightarrow 0$, using Taylor expansion, $U_{d}^{+} \sim r^{2}$ (due to the mirror symmetry $\partial_{r} U^{+}=0$ at $r=0$ ), and $W_{12}^{+} \approx r$, thus $\ell_{12}^{+}=\sqrt{W_{12}^{+}} / S^{+} \propto$ $1 / \sqrt{r}$; on the other hand, the well-known fact $\overline{u_{i}^{\prime} u_{i}^{\prime}} \rightarrow C_{i}>0$ leads to $\ell_{i i}^{+}=\sqrt{W_{i i}^{+}} / S^{+} \propto$ $1 / r$ near the centre. Hence, we obtain (2.20), where $\ell_{i j}=\ell_{i j}^{+} / R e_{\tau}$.

To obtain (2.20) from symmetry analysis, additional physical considerations are needed. As we know, when $r \rightarrow 0$, mean shear $S^{+}$is much smaller than $W_{12}^{+}$and hence can be ignored in the mean momentum equation; meanwhile, for the stress equations, production and viscous diffusion terms approach zero and can be ignored also, whilst turbulent transport, pressure-strain and dissipation are the dominant terms. Note that for $W_{12}^{+}$, dissipation is much smaller than pressure-strain and turbulent transport (Pope 2000) hence also ignored. Thus, approximate balance equations for the core layer are obtained as 
Quantifying wall turbulence: Reynolds stresses

$$
\begin{aligned}
& \frac{\partial W_{12}^{+}}{\partial r}+1 \approx 0, \\
& 0 \approx-\overline{u^{\prime} \frac{\partial p^{\prime}}{\partial \tilde{x}}}+\frac{\partial}{\partial r}\left(\frac{1}{2} \overline{u^{\prime} u^{\prime} v^{\prime}}+\right)-\frac{1}{R e_{\tau}} \overline{\left|\nabla u^{\prime}\right|^{+}}, \\
& 0 \approx-{\overline{v^{\prime}}}_{\frac{\partial p^{\prime}}{\partial r}}^{+}+\frac{\partial}{\partial r}\left(\frac{1}{2}{\overline{v^{\prime} v^{\prime} v^{\prime}}}^{+}\right)-\frac{1}{R e_{\tau}}{\overline{\left|\nabla v^{\prime}\right|^{+}}}^{2}, \\
& 0 \approx-{\overline{w^{\prime}}}_{\frac{\partial p^{\prime}}{\partial \tilde{z}}}^{+}+\frac{\partial}{\partial r}\left(\frac{1}{2}{\overline{w^{\prime} w^{\prime} v^{\prime}}}^{+}\right)-\frac{1}{R e_{\tau}}{\overline{\left|\nabla w^{\prime}\right|^{+}}}^{2}, \\
& 0 \approx \frac{1}{2}{\overline{u^{\prime}} \frac{\partial p^{\prime}}{\partial r}}^{+}-\frac{1}{2}{\overline{v^{\prime}}}_{\frac{\partial p^{\prime}}{\partial \tilde{x}}}^{+}+\frac{\partial}{\partial r}\left(\frac{1}{2}{\overline{u^{\prime} v^{\prime} v^{\prime}}}^{+}\right) \text {. }
\end{aligned}
$$

Applying (2.2) to (2.21), one obtains a particular dilation:

$$
\begin{gathered}
q_{1}=q_{2}=q_{3}=q=\overline{\lambda_{1} \lambda_{2}}, \\
\overline{\lambda_{1} \lambda_{p}}=\overline{\lambda_{2} \lambda_{p}}=\overline{\lambda_{1} \lambda_{2} \lambda_{2}}, \quad \overline{\lambda_{i} \lambda_{p}}=\overline{\lambda_{i} \lambda_{i} \lambda_{2}}=\overline{\lambda_{i} \lambda_{i}} /\left(q \lambda_{R}\right) .
\end{gathered}
$$

Therefore, the lengths are dilated as

$$
\ell_{12}^{*}=\left(\sqrt{q^{3}} / \lambda_{U}\right) \ell_{12}, \quad \ell_{i i}^{*}=\left(q \sqrt{\overline{\lambda_{i} \lambda_{i}}} / \lambda_{U}\right) \ell_{i i} .
$$

Now, we show that the result (2.23) is consistent with the expansion solution (2.20) in the core layer. Note that as $r \rightarrow 0, U_{d}^{+} \sim r^{2}$, thus $\lambda_{U}=q^{2}$. Moreover, $\overline{u_{i}^{\prime} u_{i}^{\prime}} \rightarrow C_{i}>$ 0 , which gives $\overline{\lambda_{i} \lambda_{i}}=1$. Further, with $\overline{\lambda_{1} \lambda_{2}}=q$ in $\left(2.22 a\right.$ ) (consistent with $W_{12}^{+} \sim r$ ), equation (2.23) leads to $\ell_{12}^{*}=(1 / \sqrt{q}) \ell_{12}, \ell_{i i}^{*}=(1 / q) \ell_{i i}$, and hence $\ell_{12} \sim r^{-1 / 2}, \ell_{i i} \sim r^{-1}$. Therefore, we have the following power laws for the core layer $(i=1,2,3)$ :

$$
\ell_{12}=I_{12}^{\text {core }} / \sqrt{r}, \quad \ell_{i i}=I_{i i}^{\text {core }} / r .
$$

\subsection{Homogeneous dilations in the bulk layer}

Unlike in the core layer, productions in the bulk are the dominant terms in the stress equations and hence cannot be ignored. Thus, by including $\overline{u^{\prime} v^{\prime}} \partial_{r} U^{+}$in $(2.21 b)$ and $\overline{v^{\prime} v^{\prime}} \partial_{r} U^{+}$in $(2.21 e)$, and applying (2.2) to the new balance equations, one obtains

$$
\begin{gathered}
q_{1}=q_{2}=q_{3}=q=\overline{\lambda_{1} \lambda_{2}}=\overline{\lambda_{1} \lambda_{1}}=\overline{\lambda_{2} \lambda_{2}}, \quad \lambda_{R}=1 /\left(q \lambda_{U}\right), \\
\overline{\lambda_{1} \lambda_{p}}=\overline{\lambda_{2} \lambda_{p}}=\overline{\lambda_{2} \lambda_{2} \lambda_{2}}=\overline{\lambda_{1} \lambda_{1} \lambda_{2}}=q \lambda_{U} .
\end{gathered}
$$

Therefore, the lengths are dilated as

$$
\ell_{i j}^{*}=\left(q^{3 / 2} / \lambda_{U}\right) \ell_{i j}
$$

for $i, j=1,2$. Note that dilation on $\ell_{33}$ is the same as $\ell_{i j}$ in (2.26) under the isotropic turbulence assumption.

Denote $\lambda_{U}=q^{3 / 2-\alpha}$ so that lengths obey the following dilations in the bulk flow:

$$
r^{*}=\mathrm{e}^{\varepsilon} r, \quad \ell_{i j}^{*}=\mathrm{e}^{\alpha \varepsilon} \ell_{i j} .
$$




$\begin{array}{ccccc} & \text { Sublayer } & \text { Buffer } & \text { Bulk } & \text { Core } \\ \ell_{12} & y^{3 / 2} & y^{2} & 1-r^{4} & r^{-1 / 2} \\ \ell_{11} & y & y^{3 / 2} & 1-r^{4} & r^{-1} \\ \ell_{22} & y^{2} & y^{5 / 2} & 1-r^{4} & r^{-1} \\ \ell_{33} & y & y^{2} & 1-r^{4} & r^{-1}\end{array}$

TABLE 1. A summary of the canonical scaling in the four layers of turbulent channels. Note that the $\log$ layer is contained in the bulk layer since $1-r^{4} \propto y$ as $r=1-y \rightarrow 1$ (towards the wall). For pipe flows, the scalings are the same as for channels except for $\ell_{i j} \propto 1-r^{5}$ in the bulk layer of pipes.

Now, following the second ansatz in Part 1, the differential invariants of the lengths are assumed to be constants in the bulk layer, leading to

$$
(\mathrm{d} / \mathrm{d} r) \ell_{i j}=I_{i j} r^{\alpha-1}
$$

(where $I_{i j}$ are constants). After integration with respect to $r$, equation (2.28) leads to

$$
\ell_{i j}=b_{i j}-\frac{I_{i j} r^{\alpha}}{\alpha}
$$

Furthermore, in the limit $r \rightarrow 1$ (to the wall), all lengths go to zero, then we readily obtain (for $i=1,2,3$ )

$$
\ell_{12}=I_{12}^{\prime}\left(1-r^{\alpha}\right) ; \quad \ell_{i i}=I_{i i}^{\prime}\left(1-r^{\alpha}\right) .
$$

Note that all the lengths in (2.30) asymptotes to the linear $y$ scaling (since $1-r^{\alpha}=$ $\left.1-(1-y)^{\alpha} \rightarrow \alpha y\right)$, hence matching the linear scaling (2.8) in the log layer. Also, as explained in Part 1 and Chen, Hussain \& She (2016b), $\alpha=4$ for channels and 5 for pipes.

\subsection{A summary of canonical dilation structure}

The above scalings obtained in each layer for $\ell_{12}, \ell_{11}$ and $\ell_{22}$ are summarized in table 1. Then, following exactly the same matching procedure in Part 1 (using the third ansatz and the multiplicative rule; see appendix A), we readily obtain the following multilayer formula of lengths for channels:

$$
\begin{aligned}
& \ell_{12}^{+}=c_{12} y^{+3 / 2}\left(1+\left(\frac{y^{+}}{y_{\text {sub }}^{+}}\right)^{4}\right)^{1 / 8}\left(1+\left(\frac{y^{+}}{y_{\text {buf }}^{+}}\right)^{4}\right)^{-1 / 4} \frac{1-r^{4}}{4(1-r)}\left(1+\left(\frac{r_{\text {core }}}{r}\right)^{2}\right)^{1 / 4}, \\
& \ell_{11}^{+}=c_{11} y^{+}\left(1+\left(\frac{y^{+}}{y_{\text {sub }}^{+}}\right)^{4}\right)^{1 / 8}\left(1+\left(\frac{y^{+}}{y_{\text {buf }}^{+}}\right)^{4}\right)^{-1 / 8} \frac{1-r^{4}}{4(1-r)}\left(1+\left(\frac{r_{\text {core }}}{r}\right)^{2}\right)^{1 / 2}, \\
& \ell_{22}^{+}=c_{22} y^{+2}\left(1+\left(\frac{y^{+}}{y_{\text {sub }}^{+}}\right)^{4}\right)^{1 / 8}\left(1+\left(\frac{y^{+}}{y_{\text {buf }}^{+}}\right)^{4}\right)^{-3 / 8} \frac{1-r^{4}}{4(1-r)}\left(1+\left(\frac{r_{\text {core }}}{r}\right)^{2}\right)^{1 / 2} .
\end{aligned}
$$

Note that (2.31) has been obtained in Part 1, and the lengths in the above equations (2.31)-(2.33) are the same for both channels and pipes - except for the bulk factor 
being $\left(1-r^{5}\right) / 5(1-r)$ in pipes. Here, $c_{i j}(i, j=1,2)$ are the global proportionality coefficients (represented by Kármán-like constants $\kappa_{i j}$ defined later), which may have a moderate $R e$ dependence, and are responsible for the finite $R e$-scaling for the peak magnitude of Reynolds stresses (see detailed analysis below).

The above equations therefore lead to

$$
\begin{aligned}
& \alpha_{11}=\frac{\ell_{11}^{+}}{\ell_{12}^{+}}=\frac{\sqrt{W_{11}^{+}}}{\sqrt{W_{12}^{+}}}=c_{1}\left(1+\left(\frac{y_{\text {buf }}^{+}}{y^{+}}\right)^{4}\right)^{1 / 8}\left(1+\left(\frac{r_{\text {core }}}{r}\right)^{2}\right)^{1 / 4}, \\
& \alpha_{22}=\frac{\ell_{22}^{+}}{\ell_{12}^{+}}=\frac{\sqrt{W_{22}^{+}}}{\sqrt{W_{12}^{+}}}=c_{2}\left(1+\left(\frac{y_{\text {buf }}^{+}}{y^{+}}\right)^{4}\right)^{-1 / 8}\left(1+\left(\frac{r_{\text {core }}}{r}\right)^{2}\right)^{1 / 4}
\end{aligned}
$$

where $c_{1}=c_{11} /\left(c_{12} y_{b u f}^{+1 / 2}\right)$ and $c_{2}=c_{22} y_{b u f}^{+1 / 2} / c_{12}$. Note that $\alpha_{11}$ and $\alpha_{22}$ have been introduced and studied for compressible turbulent boundary layers (Morkovin 1962; Bradshaw 1977; Zhang et al. 2012). The two ratios can be interpreted to describe the relative sizes of turbulent eddies associated with different fluctuation components, which have simple scaling properties as follows. In the near-wall region, equation (2.34) yields $\alpha_{11} \propto 1 / \sqrt{y^{+}}$as $y^{+} \rightarrow 0$, because $W_{11}^{+} \propto y^{+2}$ and $W_{12}^{+} \propto y^{+3}$. In the core layer, $W_{12}^{+} \approx r$ and $W_{11}^{+}=$const., hence $\alpha_{11} \propto 1 / \sqrt{r}$. In the overlap region, i.e. $y^{+} \gg 1$ and $r \rightarrow 1$, fluctuations are nearly isotropic; hence $\alpha_{11} \approx$ const. (an anomalous scaling modification will be introduced for the emergence of outer peak of $\overline{u^{\prime} u^{\prime}}$, as explained later). Similarly, for $\alpha_{22}$, equation (2.35) yields $\alpha_{22} \propto \sqrt{y^{+}}$as $y^{+} \rightarrow 0$, due to $v^{\prime} \propto y^{2}$; the overlap region and core layer scaling of $\alpha_{22}$ are the same as $\alpha_{11}$. Also note that the exponent 2 of the core layer factor $\left(r_{\text {core }} / r\right)^{2}$ in (2.34) and (2.35) leads to a parabolic profile for each Reynolds normal stress, i.e. $W_{i i}^{+}-W_{i i c}^{+} \propto r^{2}$, hence satisfying mirror symmetry at the centreline.

The above analysis of $\alpha_{11}$ and $\alpha_{22}$ also applies to $\alpha_{33}\left(\equiv \sqrt{W_{33}^{+} / W_{12}^{+}}\right)$. As $W_{33}^{+} \propto y^{+2}$ and $W_{12}^{+} \propto y^{+3}$ when $y^{+} \rightarrow 0$, one has $\alpha_{33} \propto 1 / \sqrt{y^{+}}$. Near the centre, $\alpha_{33} \propto 1 / \sqrt{r}$ due to a non-zero value of $W_{33}^{+}$at $r=0$. In the overlap region, the isotropic turbulence assumption implies the same scaling for all quantities, and hence a constant $\alpha_{33}$ (an anomalous scaling modification will be introduced later). Since all the scalings in these three layers are the same as those of $\alpha_{11}$, we expect that $\alpha_{33}$ takes the same functional form as $\alpha_{11}$ in (2.34). However, there is a significant difference between $W_{11}^{+}$and $W_{33}^{+}$in their balance mechanisms: the (net) production-dissipation balance of $W_{11}^{+}$occurs until above the buffer layer thickness (hence the overlap region) in contrast to the pressure-strain-dissipation balance of $W_{33}^{+}$just above the sublayer thickness. Because of this difference, the scaling transition to the overlap region occurs earlier at $y_{\text {sub }}^{+}$for $\alpha_{33}$, in contrast to at $y_{\text {buf }}^{+}$for $\alpha_{11}$. Thus, the expression for $\alpha_{33}$ is

$$
\alpha_{33}=\frac{\ell_{33}^{+}}{\ell_{12}^{+}}=\frac{\sqrt{W_{33}^{+}}}{\sqrt{W_{12}^{+}}}=c_{3}\left(1+\left(\frac{y_{\text {sub }}^{+}}{y^{+}}\right)^{4}\right)^{1 / 8}\left(1+\left(\frac{r_{\text {core }}}{r}\right)^{2}\right)^{1 / 4} .
$$

Multiplying $\alpha_{33}$ with $\ell_{12}^{+}$yields

$$
\ell_{33}^{+}=c_{33} y^{+}\left(1+\left(\frac{y^{+}}{y_{\text {sub }}^{+}}\right)^{4}\right)^{1 / 4}\left(1+\left(\frac{y^{+}}{y_{\text {buf }}^{+}}\right)^{4}\right)^{-1 / 4} \frac{1-r^{4}}{4(1-r)}\left(1+\left(\frac{r_{\text {core }}}{r}\right)^{2}\right)^{1 / 2}
$$


where $c_{33}=c_{3} c_{12} y_{s u b}^{+1 / 2}$. The local scaling of (2.37) is also summarized in table 1 . Figure $1(b)$ shows the compensated plot for all lengths divided by $1-r^{4}$, where a typical four-layer structure is clearly visible.

In the sections below, we will compare above formulae with data. It turns out that two normal fluctuation-related components, i.e. $W_{12}^{+}$and $W_{22}^{+}$(related to active motions), follow exactly the canonical four-layer description. The two other components $W_{11}^{+}$and $W_{33}^{+}$(related to inactive motions) show notable departures from the four-layer descriptions (which lead to constant $W_{11}^{+}$and $W_{33}^{+}$in the bulk), but are readily improved by the aforementioned, slight modifications of the scalings in the $\log$ layer.

\section{Reynolds shear stress $W_{12}^{+}$}

Integrating the mean momentum equation with respect to the wall distance

$$
S^{+}+W_{12}^{+}=1-y^{+} / R e_{\tau}=r
$$

and solving it yields a quadratic expression for the Reynolds shear stress $W_{12}^{+}$:

$$
W_{12}^{+}=r-S^{+}=r\left[1-2 /\left(1+\sqrt{1+4 r \ell_{12}^{+2}}\right)\right],
$$

where $\ell_{12}^{+}$is given in (2.31). According to Part 1 , the parameter $c_{12}$ in (2.31) is

$$
c_{12}=\ell_{0} /\left(y_{\text {sub }}^{+3 / 2} Z_{\text {core }}\right), \quad \ell_{0}=\kappa y_{\text {sub }}^{+2} / y_{\text {buf }}^{+}, \quad Z_{\text {core }}=\left(1+r_{\text {core }}^{2}\right)^{1 / 4},
$$

where $y_{\text {sub }}^{+}=9.7, \kappa=0.45, y_{\text {buf }}^{+}=41$ and $r_{\text {core }}=0.27$. Thus, equation (3.3) gives $\ell_{0} \approx$ $1.03, Z_{\text {core }} \approx 1.02$ and $c_{12} \approx 0.033$. Figure 2 shows that the corresponding $W_{12}^{+}$profiles compare well with data, with the departure bounded within \pm 0.01 . Note that Part 1 found $y_{\text {buf }}^{+}$and $r_{\text {core }}$ weakly dependent on $R e$ for the description of MVP; however, they are not sensitive to $R e$ for the description of $W_{12}^{+}$and hence are kept constants through this paper. Below, we discuss two interesting issues: namely, the location of maximum production, and the scaling of the $W_{12}^{+}$peak value and its location.

\subsection{Location of maximum production}

Near the wall, the mean momentum equation (3.1) leads to $S^{+}+W_{12}^{+} \approx 1$. Thus, the production $S^{+} W_{12}^{+} \approx S^{+}\left(1-S^{+}\right)$has its maximum value of $1 / 4$, located at the $y_{\max }^{+}$ where $S^{+}=W_{12}^{+}=1 / 2$. Then $\ell_{12}^{+}=\sqrt{W_{12}^{+}} / S^{+}=\sqrt{2}$ at $y_{\text {max }}^{+}$.

On the other hand, as the wall is approached, i.e. $y^{+} \rightarrow 0$, equation (2.31) asymptotes to its sublayer expression:

$$
\ell_{12}^{+}=\ell_{0}\left(y^{+} / y_{\text {sub }}^{+}\right)^{3 / 2} \approx\left(y^{+} / y_{\text {sub }}^{+}\right)^{3 / 2} .
$$

Then, letting $\ell_{12}^{+}=\sqrt{2}$ in (3.4), one has

$$
y_{\max }^{+}=\sqrt[3]{2} y_{\text {sub }}^{+} \approx 12.2
$$

which is very close to the documented value of approximately 12 (Kim, Moin \& Moser 1987; Pope 2000). The result $y_{\max }^{+} \approx 12.2$ can be inverted to measure the sublayer thickness $y_{\text {sub }}^{+} \approx 9.7$. 
(a)

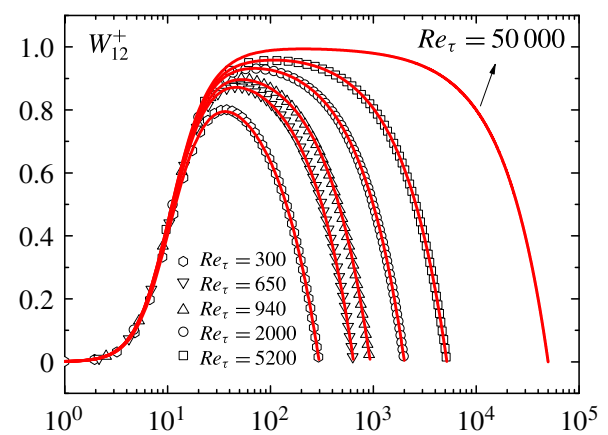

(c)

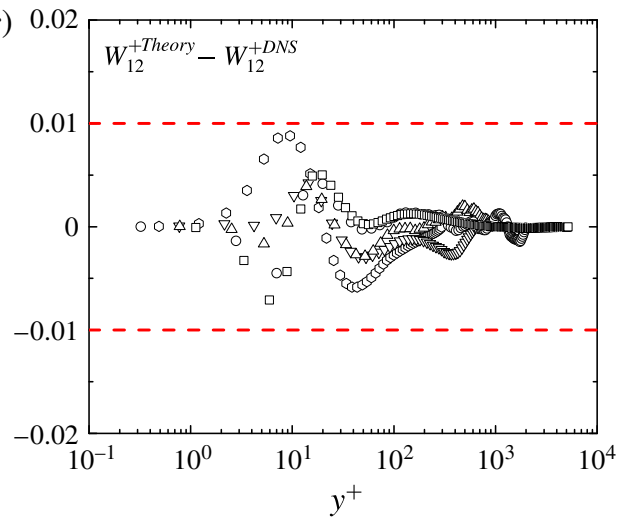

(b)

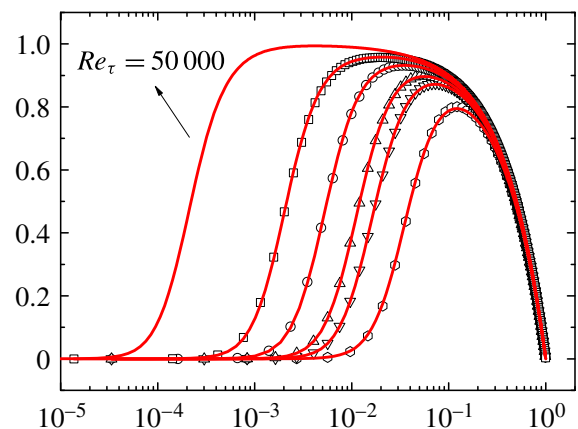

(d)

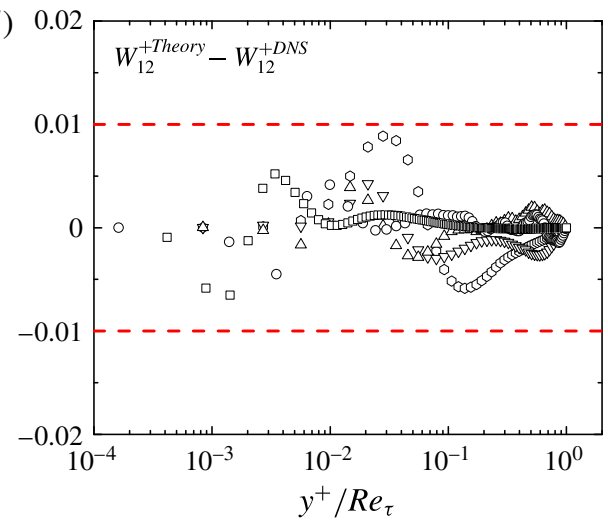

FIGURE 2. (Colour online) Reynolds shear stress profiles by (3.2) (solid lines) compared with DNS data (symbols) for channel flows in coordinates $y^{+}(a)$ and $y^{+} / \operatorname{Re}_{\tau}(b)$. The departure between data and our theory (i.e. $W_{12}^{+ \text {Theory }}-W_{12}^{+D N S}$ ) is shown in $(c, d)$, which is bounded within \pm 0.01 . DNS channel data from Iwamoto, Suzuki \& Kasagi (2002) for $R e_{\tau}=300,650$, Hoyas \& Jimenez (2006) for $R e_{\tau}=940,2000$; Lee \& Moser (2015) at $R e_{\tau}=5200$. Also included are the prediction of $W_{12}^{+}$for $R e_{\tau}=50000$, awaiting future verification.

Note that $W_{12}^{+}$predicted by (3.4) also agrees with data in most of the flow domain (not shown here) - except for the buffer layer. This is because: (1) for the outer region, equation (3.4) yields $\ell_{12}^{+} \gg 1$, which leads to $W_{12}^{+} \approx r-$ a good approximation in the entire outer flow; (2) for the inner region, equation (3.4) gives an accurate description of $W_{12}^{+}$in the viscous sublayer. However, for the buffer layer, the simple sublayer formula (3.4) is insufficient and needs to be extended to predict how $W_{12}^{+}$approaches its peak value, addressed below.

\subsection{Scaling for the peak of Reynolds shear stress}

To determine the peak of $W_{12}$, equation (3.2) is approximated to $W_{12}^{+} \approx r-\sqrt{r} / \ell_{12}^{+}$ (since $\ell_{12}^{+} \gg 1$ as $y^{+} \gg y_{\text {sub }}^{+}$). Further, with $r=1-y^{+} / R e_{\tau}$, we have:

$$
W_{12}^{+} \approx 1-y^{+} / R e_{\tau}-1 / \ell_{12}^{+}
$$

(the $1 / R e_{\tau}$ term in $\sqrt{r}$ is small and ignored). Letting $\partial_{y^{+}} W_{12}^{+}=0$ using (3.6), one has the following relation at the peak location (i.e. $y_{12 p}^{+}$) of $W_{12}^{+}$:

$$
R e_{\tau}^{-1}=\dot{\ell}_{12}^{+} / \ell_{12}^{+2} \text {. }
$$

Here, the superscript dot indicates derivative with respect to $y^{+}$. 

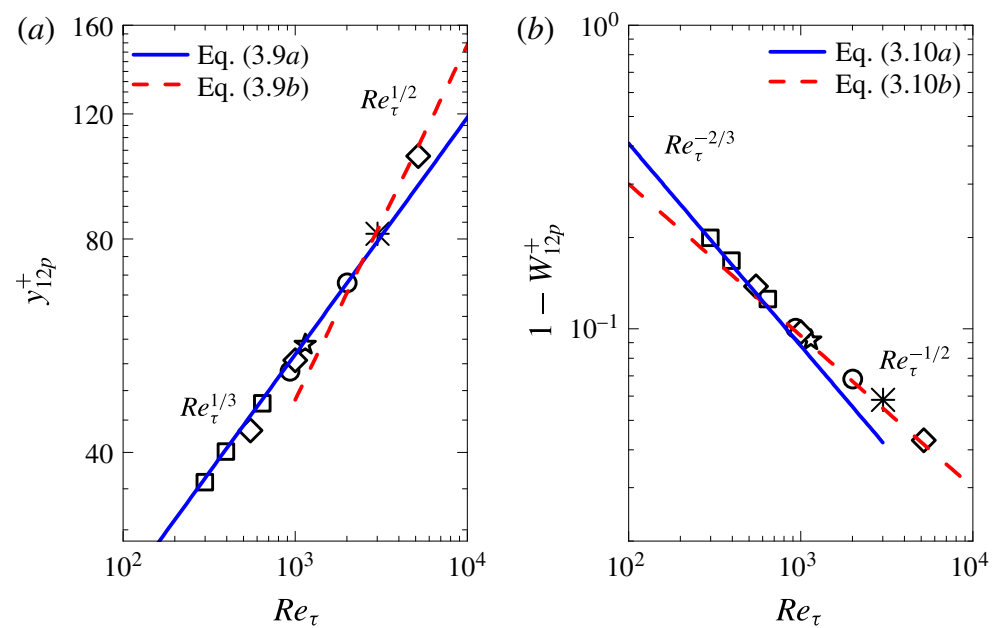

FIGURE 3. (Colour online) Scaling for the peak of Reynolds shear stress: (a) location; (b) magnitude defect. Solid lines denote $(3.9 a)$ and (3.10a) for low $R e$, and dashed lines denote $(3.9 b)$ and $(3.10 b)$ for high $R e$. Symbols are DNS data: squares from Iwamoto et al. (2002) for $R e_{\tau}=300,400$ and 650 (channel); circles from Hoyas \& Jimenez (2006) for $\operatorname{Re}_{\tau}=940$ and 2000 (channel); stars from Wu \& Moin (2008) for $\operatorname{Re}_{\tau}=1142$ (pipe); cross from Ahn et al. (2015) for $R e_{\tau}=3008$ (pipe); and diamonds from Lee \& Moser (2015) for $R e_{\tau}=550,1000$ and 5200 (channel).

By substituting the stress length (2.31) into (3.7) we can solve for $y_{12 p}^{+}$, which is located in the buffer layer for small $R e$, but in the log layer for large $R e$. Since $\ell_{12}^{+}$ from (2.31) has the following two simple asymptotes:

$$
\begin{gathered}
\ell_{12}^{+} \approx\left(\kappa / y_{\text {buf }}^{+}\right) y^{+2}, \quad \text { for } y_{\text {sub }}^{+} \ll y_{12 p}^{+} \lesssim y_{\text {buf }}^{+}, \\
\ell_{12}^{+} \approx \kappa y^{+}, \quad \text { for } y_{\text {buf }}^{+} \ll y_{12 p}^{+} \ll R e_{\tau},
\end{gathered}
$$

then $y_{12 p}^{+}$- obtained by substituting (3.8) into (3.7) - has the following two scalings:

$$
\begin{gathered}
y_{12 p}^{+}=\operatorname{Re}_{\tau}^{1 / 3}\left(2 y_{\text {buf }}^{+} / \kappa\right)^{1 / 3} \approx 5.7 \operatorname{Re}_{\tau}^{1 / 3}, \\
y_{12 p}^{+}=\operatorname{Re}_{\tau}^{1 / 2} / \sqrt{\kappa} \approx 1.5 \operatorname{Re}_{\tau}^{1 / 2} .
\end{gathered}
$$

The $R e_{\tau}^{1 / 2}$ scaling has been found before (Sreenivasan 1988; Sreenivasan \& Sahay 1997; Klewicki et al. 2012; Lee \& Moser 2015), but the $R e_{\tau}^{1 / 3}$ scaling for small $R e$ is new. The latter $R e_{\tau}^{1 / 3}$ scaling is very close to the $R e_{\tau}^{0.372}$ scaling found by Ahn, Lee \& Sung (2017) from DNS channel and pipe data. Figure 3(a) shows that these two scalings are in good agreement with data. Note that the scaling transition from $R e_{\tau}^{1 / 3}$ to $R e_{\tau}^{1 / 2}$ - which occurs around $R e_{\tau}=4 \kappa y_{\text {buf }}^{+2} \approx 3000$ obtained by equalling (3.9a) with $(3.9 b)$ - is not sharp.

Also, the small departure of the peak value of $W_{12}^{+}$from unity shows a scaling transition (figure 3b), explained as follows. Substituting (3.8) and (3.9) back into (3.6) yields the departure:

$$
\begin{gathered}
1-W_{12 p}^{+}=3\left(y_{b u f}^{+} / 4 \kappa\right)^{1 / 3} \operatorname{Re}_{\tau}^{-2 / 3} \approx 8.5 \operatorname{Re}_{\tau}^{-2 / 3}, \\
1-W_{12 p}^{+}=(2 / \sqrt{\kappa}) \operatorname{Re}_{\tau}^{-1 / 2} \approx 3.0 \operatorname{Re}_{\tau}^{-1 / 2} .
\end{gathered}
$$


(a)

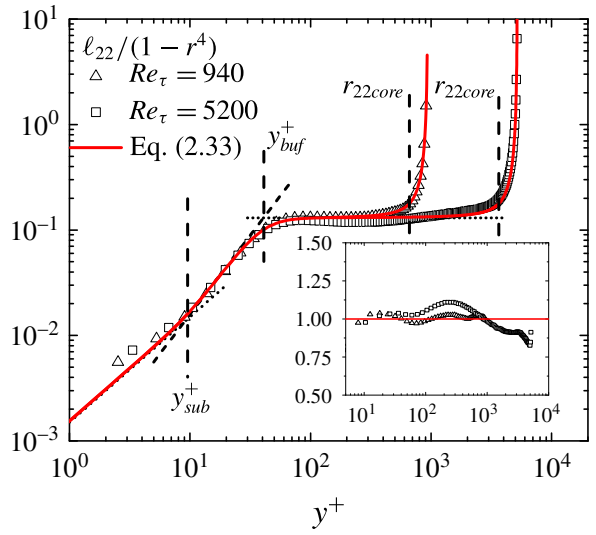

(b)

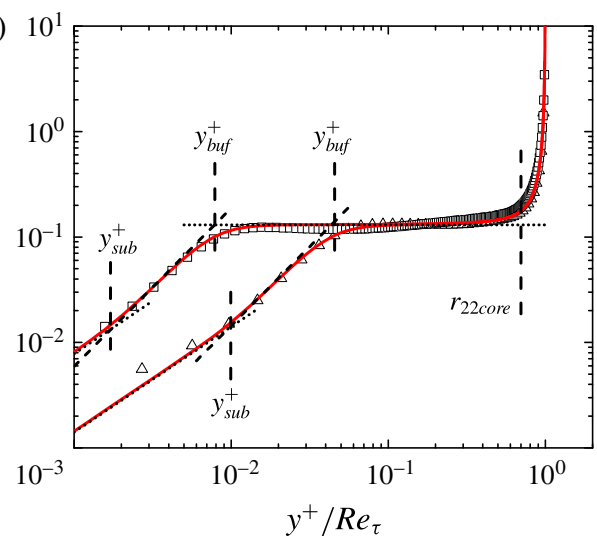

FIGURE 4. (Colour online) Validation of (2.33) against DNS data for $\ell_{22}^{+}$as a function of $y^{+}(a)$ and $y^{+} / \operatorname{Re}_{\tau}(b)$ with $y_{\text {sub }}^{+}=9.7, y_{\text {buf }}^{+}=41$ (the same as for $\ell_{12}^{+}$), $\kappa_{22}=0.52$ and $r_{22 \text { core }}=0.3$. Inset of $(a)$ shows the compensated plot of $\ell_{22}^{\text {Theory }} / \ell_{22}^{D N S}$ using (2.33) and DNS data, which are close to the unity in almost the entire flow region.

These scalings agree well with data (figure $3 b$ ). Note that (3.10) also predicts the $R e$ dependence of $B_{12}$ in (1.1) since $B_{12}=W_{12 p}^{+}$(the plateau of $W_{12}^{+}$in the log layer is well represented by its peak value).

\section{Wall-normal Reynolds stress $W_{22}^{+}$}

The wall-normal Reynolds stress $W_{22}$ (or the wall-normal fluctuation intensity) is related to the Reynolds shear stress $W_{12}$ by

$$
W_{22}^{+}=W_{12}^{+} \ell_{22}^{+2} / \ell_{12}^{+2},
$$

which can then be obtained by the formulae developed in the above sections (i.e. (3.2), (2.31) and (2.33)). A direct test of the theoretical $\ell_{22}^{+}$at two $R e$ values $\left(R e_{\tau}=940\right.$ and $5200)$ is shown in figure 4 , where the agreement is very good. Note that the parameter $c_{22}$ (or equivalently $\kappa_{22}$ introduced below) is easily measured by the peak value of $W_{22}^{+}$from DNS data. Similarly, the core layer thickness $r_{\text {core }}$ of $\ell_{22}^{+}$in $(2.33)-$ now denoted as $r_{22 \text { core }}$ - is measured to be 0.3 from the centreline value of $W_{22}^{+}$, which is $10 \%$ larger than $r_{\text {core }}=0.27$ for $\ell_{12}^{+}$(explained later). With these two new parameters, the resulting $W_{22}^{+}$profiles agree well with data (figure 5).

\subsection{Measurement of $\kappa_{22}$}

Let us consider the scaling of the peak value of $W_{22}^{+}$for asymptotically large $R e$. Let $c_{22}=c_{22}^{\prime} / \sqrt{1+r_{22 \text { core }}^{2}}$ in (2.33) so that $\ell_{22}^{+} \approx c_{22}^{\prime} y^{+2}$ for $y^{+} \rightarrow 0$. On the other hand, for $y^{+} \gg y_{b u f}^{+}$, we obtain the linear scalings: $\ell_{12}^{+} \approx \kappa y^{+}$and $\ell_{22}^{+} \approx \kappa_{22} y^{+}$, where $\kappa_{22}=$ $c_{22}^{\prime} y_{\text {buf }}^{+3 / 2} / y_{\text {sub }}^{+1 / 2}$. Accordingly, $W_{22}^{+} \approx W_{12}^{+}\left(\kappa_{22} / \kappa\right)^{2}$. Thus, its peak value is

$$
W_{22 p}^{+} \approx W_{12 p}^{+} \kappa_{22}^{2} / \kappa^{2} .
$$

Therefore,

$$
\kappa_{22} \approx \kappa \sqrt{W_{22 p}^{+} / W_{12 p}^{+}} .
$$


(a)

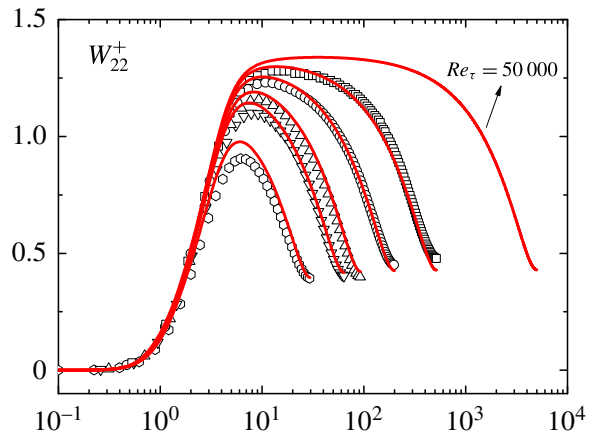

(c)

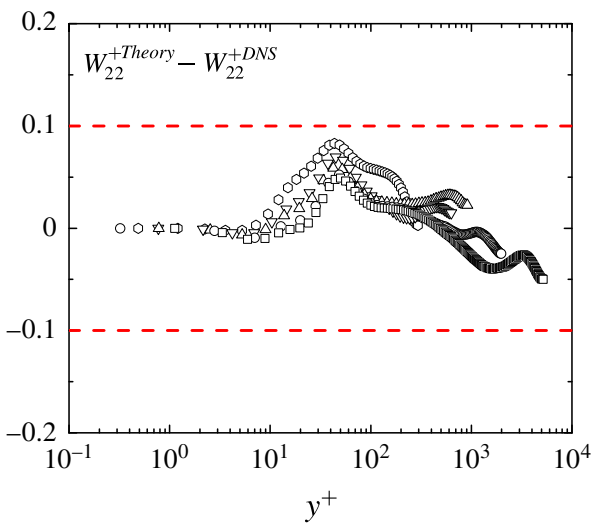

(b)

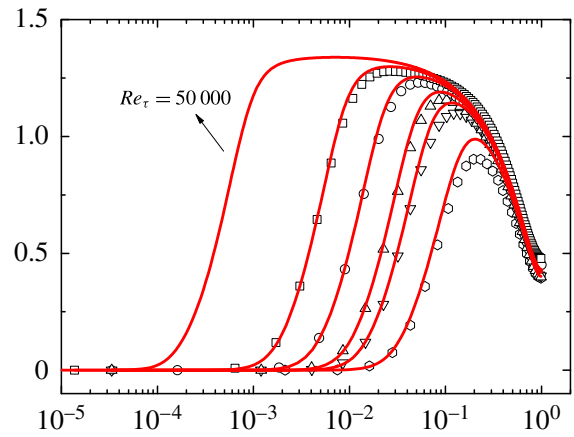

(d)

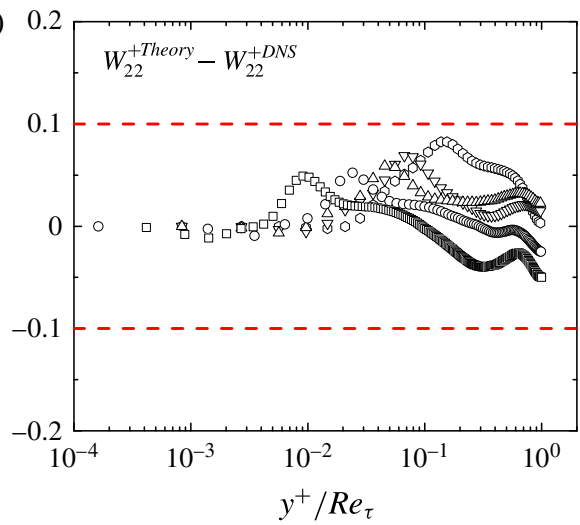

FIgURE 5. (Colour online) Wall-normal Reynolds stress profiles by (4.1) compared with data. Also included are predictions at $R e_{\tau}=50000$. The departure between data and our theory (i.e. $W_{22}^{+ \text {Theory }}-W_{22}^{+D N S}$ ) is shown in $(c, d)$, which is bounded within \pm 0.1 . For legend, see figure 2.

This yields a method to measure the new constant $\kappa_{22}$. For $R e_{\tau}=5200$, with $\kappa=0.45$, we predict $W_{12 p}^{+} \approx 0.96$ from $(3.10 b)$. Then, with the measured $W_{22 p}^{+} \approx 1.29$ from data at $R e_{\tau}=5200$ (Lee \& Moser 2015 ), we obtain $\kappa_{22} \approx 0.52 \approx 1.16 \kappa$.

Note that according to $W_{22}^{+} \approx W_{12}^{+}\left(\kappa_{22} / \kappa\right)^{2}, W_{22}^{+}$should reach its peak at the same location as the peak of $W_{12}^{+}$for large $R e$; i.e. $y_{22 p}^{+}=\sqrt{R e_{\tau} / \kappa}$. However, $R e$ for the current data is not high enough to show such a scaling, and higher-Re data are needed to test it.

\subsection{Measurement of $r_{22 \text { core }}$}

Near the centreline (as $r \rightarrow 0), \ell_{12}^{+} \approx(\kappa / 4)\left(1+r_{\text {core }}^{2} / r^{2}\right)^{1 / 4}$ from $(2.31)$, and $\ell_{22}^{+} \approx$ $\left(\kappa_{22} / 4\right)\left(1+r_{22 \text { core }}^{2} / r^{2}\right)^{1 / 2}$ from (2.33). Further, with $W_{12}^{+} \approx r$, the centreline value is given as

$$
W_{22 c}^{+}=W_{12}^{+}\left(\ell_{22}^{+} / \ell_{12}^{+}\right)^{2} \approx \kappa_{22}^{2} r_{22 \text { core }}^{2} /\left(\kappa^{2} r_{\text {core }}\right),
$$

which leads to

$$
r_{22 \text { core }} \approx\left(W_{22 c}^{+} r_{\text {core }}\right)^{1 / 2}\left(\kappa / \kappa_{22}\right) .
$$

Thus, with $r_{\text {core }}=0.27, \kappa=0.45$, as well as $\kappa_{22} \approx 0.52$ and $W_{22 c}^{+} \approx 0.47$ from DNS data (Lee \& Moser 2015), we have $r_{22 \text { core }} \approx 0.3$. This also results in $\kappa_{22} \approx 0.78 W_{22 c}^{+1 / 2}$. 

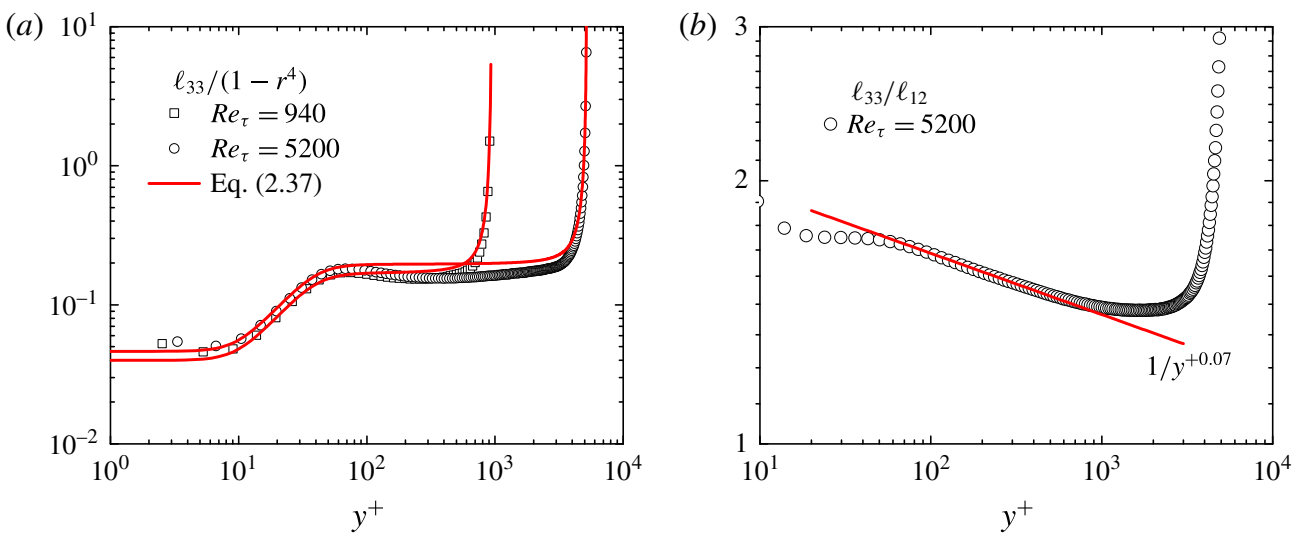

FIGURE 6. (Colour online) Canonical four-layer $\ell_{33}$ of (2.37) compared with data: $(a)$ compensated plot of $\ell_{33}$ divided by $1-r^{4} ;(b)$ compensated plot of $\ell_{33}$ divided by $\ell_{12}$ showing an anomalous scaling in the bulk flow region. As explained in the text, the anomalous scaling exponent 0.07 results from (5.5). Symbols denote DNS channel data at $R e_{\tau}=940$ (Hoyas \& Jimenez 2006) and $R e_{\tau}=5200$ (Lee \& Moser 2015).

Note that if one imposes $r_{22 \text { core }}=r_{\text {core }}=0.27$, the resulting $W_{22 c}^{+}$from (4.4) would underpredict the data by $20 \%$ at $R e_{\tau}=5200$. For the smallest $\operatorname{Re}_{\tau}(=300)$, DNS data shows $W_{22 c}^{+} \approx 0.39$ (Iwamoto et al. 2002), so that (4.5) leads to $r_{22 \text { core }} \approx 0.28$. Therefore, $r_{22 \text { core }}$ varies mildly with increasing $\operatorname{Re}_{\tau}$ (for a moderate $\operatorname{Re}_{\tau}$ range).

Substituting $\kappa_{22} \approx 0.52$ and $r_{22 \text { core }} \approx 0.3$ into $(2.33)$, where $c_{22}=\kappa_{22} y_{\text {sub }}^{+1 / 2} /\left[y_{\text {buf }}^{+3}(1+\right.$ $\left.\left.r_{22 \text { core }}^{2}\right)\right]^{1 / 2}$, the resulting $W_{22}^{+}$profiles from (4.1) are shown in figure 5 . The agreement is quite good, with the departure bounded within \pm 0.1 . Note that for the smallest $\operatorname{Re}_{\tau}(=300)$, there is a slight overestimation of the peak magnitude. This small departure can be easily rectified by $\kappa_{22} \approx 0.50$ at $R e_{\tau}=300$; hence $\kappa_{22}$ slightly changes for moderate $R e$.

\section{Spanwise Reynolds stress $W_{33}^{+}$}

The spanwise Reynolds stress $W_{33}$ is related to the Reynolds shear stress $W_{12}$ by

$$
W_{33}^{+}=W_{12}^{+} \ell_{33}^{+2} / \ell_{12}^{+2} \text {. }
$$

In (2.37), all the layer thicknesses of $\ell_{33}$ are the same as those of $\ell_{12}$; then $c_{33}$ is the only free parameter. Again, let $c_{33}=c_{33}^{\prime} / \sqrt{1+r_{\text {core }}^{2}}$ so that $\ell_{33}^{+} \approx c_{33}^{\prime} y^{+}$as $y^{+} \rightarrow 0$ (or $r \rightarrow 1)$, and $c_{33}^{\prime}=\kappa_{33} y_{\text {sub }}^{+} / y_{\text {buf }}^{+}$so that $\ell_{33}^{+} \approx \kappa_{33} y^{+}$as $y^{+} \gg y_{\text {buf }}^{+}$. Comparison of $\ell_{33}^{+}$in (2.37) with data at two values of $\operatorname{Re}\left(R e_{\tau}=940\right.$ and 5200) is shown in figure 6(a), which shows good agreement with data in the near-wall and core regions, but reveals notable differences in the bulk layer. The reason is analysed below.

\subsection{Anomalous scaling and invariant peak location}

Note that the deviation in figure $6(a)$ occurs in the log layer where $\ell_{33}^{+} \approx \kappa_{33} y^{+}$. However, as shown by the ratio $\alpha_{33}=\ell_{33}^{+} / \ell_{12}^{+}$(see figure $6 b$ ), the linear scaling of $\ell_{33}^{+}$ is invalid since $\alpha_{33} \propto 1 / y^{+0.07}$ - otherwise $\alpha_{33}$ would be a constant equalling $\kappa_{33} / \kappa$. This deviation thus suggests an anomalous scaling modification of $\ell_{33}$ - via a change of $\left(1+\left(y^{+} / y_{\text {buf }}^{+}\right)^{4}\right)^{-1 / 4}$ in $(2.37)$ to $\left(1+\left(y^{+} / y_{\text {buf }}^{+}\right)^{4}\right)^{-(1+\gamma) / 4}-$ so that $\ell_{33}^{+} \propto y^{+(1-\gamma)}$, and 
hence $\alpha_{33} \propto 1 / y^{+\gamma}$ in the log layer $\left(y^{+} \gg y_{b u f}^{+}\right)$. In other words, $\ell_{33}$ is modified to

$$
\ell_{33}^{\prime}=c_{33} y^{+}\left(1+\left(\frac{y^{+}}{y_{\text {sub }}^{+}}\right)^{4}\right)^{1 / 4}\left(1+\left(\frac{y^{+}}{y_{\text {buf }}^{+}}\right)^{4}\right)^{-(1+\gamma) / 4} \frac{1-r^{4}}{4(1-r)}\left(1+\left(\frac{r_{\text {core }}}{r}\right)^{2}\right)^{1 / 2}
$$

and hence

$$
W_{33}^{+}=W_{12}^{+} \ell_{33}^{\prime 2} / \ell_{12}^{+2}
$$

Here, $\gamma$ is the so-called anomalous scaling exponent, and its value 0.07 - shown in figure $6(b)$ - can be theoretically determined by the peak location of $W_{33}^{+}$. Note that if $\gamma=0$ (i.e. no anomalous scaling), $W_{33}^{+} \approx W_{12}^{+}\left(\kappa_{33} / \kappa\right)^{2}$ in the log layer, and thus the peak location of $W_{33}^{+}$(i.e. $y_{33 p}^{+}$) should follow that of $W_{12}^{+}$(i.e. $y_{12 p}^{+}$) - both $y_{33 p}^{+}$and $y_{12 p}^{+}$increasing rapidly with increasing $R e$. However, a careful examination of DNS data (from $R e_{\tau}=650$ to 5200 ) shows that $y_{33 p}^{+}$remains almost constant, i.e. $y_{33 p}^{+} \approx 46$, in sharp contrast to increasing $y_{12 p}^{+}$in figure 3 . This constancy of $y_{33 p}^{+}$is actually due to the existence of the anomalous scaling $\gamma \neq 0$, and enables the determination of $\gamma \approx 0.07$, as below.

In fact, for $y^{+} \simeq y_{\text {buf }}^{+}$where $y_{33 p}^{+}$is located, we have $\ell_{12}^{+} \approx\left(\kappa y^{+2} / y_{\text {buf }}^{+}\right)[1+$ $\left.\left(y^{+} / y_{\text {buf }}^{+}\right)^{4}\right]^{-1 / 4}$ from (2.31), and hence an approximation of $W_{12}^{+}$is obtained from (3.6), i.e. $W_{12}^{+} \approx 1-y^{+} / R e_{\tau}-y_{b u f}^{+} /\left(\kappa y^{+2}\right)$ (note that the $\left(y^{+} / y_{b u f}^{+}\right)^{4}$ term is very small for $W_{12}^{+}$and hence ignored). Similarly, $\ell_{33}^{\prime} \approx\left(\kappa_{33} y^{+2} / y_{\text {buf }}^{+}\right)\left[1+\left(y^{+} / y_{\text {buf }}^{+}\right)^{4}\right]^{-(1+\gamma) / 4}$ from (5.2), and thus we have

$$
W_{33}^{+}=W_{12}^{+}\left(\ell_{33}^{\prime} / \ell_{12}^{+}\right)^{2} \approx\left(\kappa_{33} / \kappa\right)^{2}\left(1-\frac{y^{+}}{R e_{\tau}}-\frac{y_{\text {buf }}^{+}}{\kappa y^{+2}}\right)\left(1+\left(\frac{y^{+}}{y_{\text {buf }}^{+}}\right)^{4}\right)^{-\gamma / 2} .
$$

Then, solving $\partial_{y^{+}} W_{33}^{+}=0$ at $y_{33 p}^{+}$using (5.4) yields the following result:

$$
\gamma=\frac{\left[2 y_{b u f}^{+} /\left(\kappa y_{33 p}^{+3}\right)-1 / R e_{\tau}\right]\left(y_{b u f}^{+4}+y_{33 p}^{+4}\right)}{2 y_{33 p}^{+3}\left[1-y_{33 p}^{+} / R e_{\tau}-y_{b u f}^{+} /\left(\kappa y_{33 p}^{+2}\right)\right]} \approx 0.05 \sigma^{-2}\left(1+\sigma^{-4}\right),
$$

where $\sigma=y_{33 p}^{+} / y_{b u f}^{+}$, and $y_{b u f}^{+}=41$ and $\kappa=0.45$ are substituted in (and the $1 / \operatorname{Re}_{\tau}$ term is small and ignored). As explained before, if $y_{33 p}^{+}$follows the scaling of $y_{12 p}^{+}$, then $\sigma=\sqrt{R e_{\tau} / \kappa} / y_{\text {buf }}^{+}$, and (5.5) leads to $\gamma \approx 0.05 \kappa^{2} y_{\text {buf }}^{+2} / R e_{\tau} \rightarrow 0$ (e.g. $\gamma \approx 0.003$ at $R e_{\tau}=5200$ ), hence indicating no anomalous scaling. In contrast, DNS data show an invariant peak location $y_{33 p}^{+} \approx 46$, then $\sigma \approx 1.12$, and (5.5) yields a constant $\gamma \approx 0.07$ the value shown in figure $6(b)$. In order words, the invariant peak location leads to the existence of the anomalous scaling. It would be intriguing to test if $\gamma=0.07$ remains invariant at higher $R e$ values.

Now, the only unknown parameter in (5.2) is $c_{33}$, which is $c_{33}=y_{s u b}^{+} \kappa_{33} /$ $\left(\sqrt{1+r_{\text {core }}^{2}} y_{\text {buf }}^{+}\right)$. Below, $\kappa_{33}$ is determined, hence yielding $c_{33}$.

\subsection{Scaling of $\kappa_{33}$ and of the $W_{33}^{+}$peak value}

Note that for $y^{+} \gg y_{b u f}^{+}, W_{12}^{+} \approx r$, then (5.3) leads to a simple expression for the outer profile of $W_{33}^{+}$:

$$
W_{33 \text { Outer }}^{+} \approx r \ell_{33}^{\prime 2} / \ell_{12}^{+2} \approx\left(\frac{\kappa_{33}}{\kappa}\right)^{2}\left(\frac{y_{\text {buf }}^{+}}{y^{+}}\right)^{2 \gamma}\left(\frac{r^{2}+r_{\text {core }}^{2}}{1+r_{\text {core }}^{2}}\right)^{1 / 2} .
$$



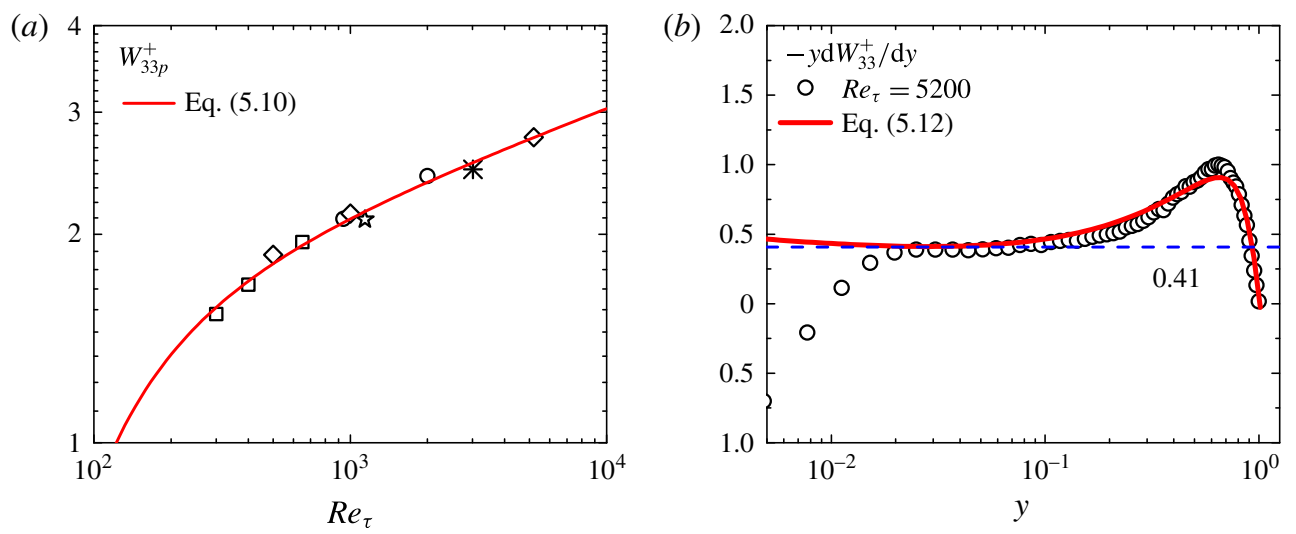

FIgURE 7. (Colour online) (a) Peak value $W_{33 p}^{+}$of DNS data compared with prediction by (5.10) (line) showing good agreement. Symbols are the same as in figure 3. (b) Diagnostic function $\Gamma_{33}=-y \mathrm{~d} W_{33}^{+} / \mathrm{d} y$ showing an approximate plateau $\Gamma_{33}=0.41 \pm 0.04$ for $0.02 \leqslant$ $y \leqslant 0.14$. Symbols are DNS data at $R e_{\tau}=5200$ (Lee \& Moser 2015). Solid line indicates (5.12), whose minimum value $\Gamma_{33 \min } \approx 0.41$ obtained in $(5.15)$ - dashed line - represents well the plateau value.

According to Townsend's $R e$-similarity hypothesis (Townsend 1976) (namely, mean properties in the outer flow region are self-similar, independent of viscous effects, and hence $R e$ ), one should expect that $W_{33 \text { Outer }}^{+}$depends only on $r$, with no dependence on $R e$. Then, requiring a zero exponent of $R e_{\tau}$ for $W_{33 \text { outer }}^{+}$in (5.6), we readily obtain $\kappa_{33} \propto \operatorname{Re}_{\tau}^{\gamma}$.

We denote $\kappa_{33}=\kappa_{33}^{\prime} R e_{\tau}^{\gamma}$, where $\kappa_{33}^{\prime}$ is a $R e$-independent constant, which can be measured by $W_{33}^{+}$at the centreline. Letting $r=0$, equation (5.6) becomes

$$
W_{33 c}^{+} \approx\left(\kappa_{33}^{\prime} / \kappa\right)^{2} y_{\text {buf }}^{+2 \gamma} r_{\text {core }} / \sqrt{1+r_{\text {core }}^{2}} \text {. }
$$

Therefore,

$$
\kappa_{33}^{\prime}=\kappa W_{33 c}^{+1 / 2}\left(1+r_{\text {core }}^{2}\right)^{1 / 4} /\left(y_{\text {buf }}^{+\gamma} r_{\text {core }}^{1 / 2}\right) .
$$

Using $\kappa=0.45, y_{\text {buf }}^{+}=41, r_{\text {core }}=0.27, \gamma=0.07$ and the centreline value $W_{33 c}^{+} \approx 0.4$ by current DNS data (Hoyas \& Jimenez 2006; Lee \& Moser 2015), we thus have $\kappa_{33}^{\prime} \approx 0.43$, and hence

$$
\kappa_{33}=\kappa_{33}^{\prime} R e_{\tau}^{\gamma} \approx 0.43 R e_{\tau}^{0.07} .
$$

Furthermore, substituting $\kappa=0.45, y_{\text {buf }}^{+}=41, \gamma=0.07, y^{+}=y_{33 p}^{+}=\sigma y_{\text {buf }}^{+}$(with $\sigma=$ 1.12) and (5.9) into (5.4), one readily obtains the peak value of $W_{33}^{+}$as

$$
\begin{aligned}
W_{33 p}^{+} & =\left(\kappa_{33}^{\prime} / \kappa\right)^{2} \operatorname{Re}_{\tau}^{2 \gamma}\left(1+\sigma^{4}\right)^{-\gamma / 2}\left[1-41 \sigma / \operatorname{Re}_{\tau}-1 /\left(41 \kappa \sigma^{2}\right)\right] \\
& \approx 0.84 \operatorname{Re}_{\tau}^{0.14}\left(1-48 / \operatorname{Re}_{\tau}\right) .
\end{aligned}
$$

To emphasize, in (5.10), $\kappa_{33}^{\prime}$ only affects the magnitude of $W_{33 p}^{+}$, while the $R e_{\tau}$ scaling of $W_{33 p}^{+}$is due to the anomalous scaling exponent $\gamma$. Figure $7(a)$ shows that $(5.10)$ agrees well with the data. Note that the downward curve in the figure $7(a)$ is due to the $48 / R e_{\tau}$ term in (5.10), which becomes negligible at large $R e$, when $W_{33 p}^{+} \approx$ $0.84 R e_{\tau}^{0.14}$. Thus, although the peak location $y_{33 p}^{+}$is invariant, the peak value $W_{33 p}^{+}$ increases with increasing $R e$. 


\subsection{Townsend's log profile for $W_{33}^{+}$}

Note that the outer profile (5.6) has a power-law asymptote towards the wall, i.e.

$$
W_{33}^{+} \approx y_{\text {buf }}^{+2 \gamma}\left(\kappa_{33}^{\prime} / \kappa\right)^{2} / y^{2 \gamma}, \quad y=1-r .
$$

This power law is conceptually different from the Townsend's log law (Townsend 1976) in (1.4). Reminiscent of the debate on the mean velocity scaling (Barenblatt 1993), a new debate between the power law and log law on the $W_{33}^{+}$scaling may arise. However, as both (5.11) and (1.4) represent data in limited but different flow domains, such a debate is really not meaningful and may not be resolved due to their unspecified $y$-ranges. Here, since we already have a global expression (5.6) which extends (5.11) to the centreline and describes $W_{33}^{+}$for the entire outer flow domain, it is more interesting to see if the present formalism (5.6) can yield the log law with its two coefficients quantitatively determined.

This is achieved via the diagnostic function of the log profile, $\Gamma_{33}=-y d_{y} W_{33}^{+}$, which is calculated using (5.6) as

$$
\Gamma_{33}=\frac{y_{\text {buf }}^{+2 \gamma}\left(\kappa_{33}^{\prime} / \kappa\right)^{2}}{\sqrt{1+r_{\text {core }}^{2}}}\left(\frac{2 \gamma \sqrt{(1-y)^{2}+r_{\text {core }}^{2}}}{y^{2 \gamma}}+\frac{(1-y) y^{1-2 \gamma}}{\sqrt{(1-y)^{2}+r_{\text {core }}^{2}}}\right) \text {. }
$$

Note that as $y \ll 1$ in the log region, equation (5.12) is further approximated to

$$
\Gamma_{33} \approx \frac{y_{\text {buf }}^{+2 \gamma}\left(\kappa_{33}^{\prime} / \kappa\right)^{2}}{\sqrt{1+r_{\text {core }}^{2}}} y^{-2 \gamma}\left(2 \gamma \sqrt{1+r_{\text {core }}^{2}}+\frac{y}{\sqrt{1+r_{\text {core }}^{2}}}\right) .
$$

Solving $\mathrm{d} \Gamma_{33} / \mathrm{d} y=0$ using (5.13), we get the $y$-location of the minimum $\Gamma_{33}$, i.e.

$$
y_{33 \min } \approx\left(1+r_{\text {core }}^{2}\right)(2 \gamma)^{2} \approx 0.021,
$$

and the corresponding minimum value $\Gamma_{33 \min }$ is

$$
\Gamma_{33 \min } \approx 2 \gamma(1+2 \gamma) y_{\text {buf }}^{+2 \gamma}\left(\kappa_{33}^{\prime} / \kappa\right)^{2}\left(1-y_{33 \min }\right) y_{33 \min }^{-2 \gamma} \approx 0.412
$$

This calculation shows that physical quantities such as $y_{b u f}^{+}, \kappa_{33}^{\prime}, \kappa, \gamma$ and $r_{\text {core }}$ contribute to the slope of the log law. It is interesting to observe that both $y_{33 \mathrm{~min}}$ and $\Gamma_{33 \min }$ (i.e. log-law slope) are independent of $R e$. Note that this $R e$-independent value is meaningful only in the log layer because of (5.6), which requires that $y_{33 \min }^{+}>y_{\text {buf }}^{+}(=41)$. This implies that only for $R e_{\tau}>y_{\text {buf }}^{+} / y_{33 \min } \approx 2000$ does the log-law behaviour of $W_{33}^{+}$become notable, as the data confirm (see figure $8 b$ ). Figure $7(b)$ shows that data agree well with our analytic expressions (5.12), (5.14) and (5.15); in particular, a plateau $\Gamma_{33}=0.41 \pm 0.04$ appears in figure $7(b)$ in the range $0.018 \leqslant y \leqslant 0.14$, very close to our prediction of $\Gamma_{33 \min }=0.412$. Then, it is demonstrated that (5.6) indeed implies an (locally approximate) log law with its slope $A_{33}=\Gamma_{33 \min } \approx 0.41$.

Furthermore, the additive constant $B_{33}$ can also be estimated by matching (5.6) with (1.4) at the position $y=y_{33 \mathrm{~min}}$. That is, substituting $y=y_{33 \Gamma}=0.021$ into (5.6) yields $W_{33 \Gamma}^{+} \approx 2.58$, and hence the additive constant $B_{33}=W_{33 \Gamma}^{+}+A_{33} \ln y_{33 \Gamma}$. Therefore, equation (5.6) theoretically leads to

$$
A_{33}=\Gamma_{33 \min } \approx 0.41, \quad B_{33}=W_{33 \Gamma}^{+}+A_{33} \ln y_{33 \Gamma} \approx 1.00
$$


(a)

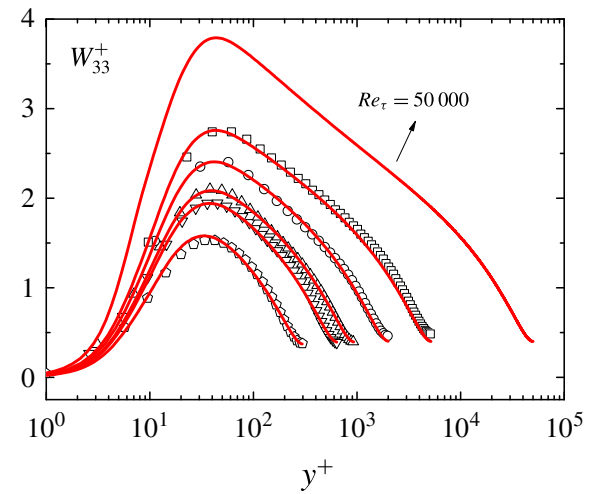

(c)

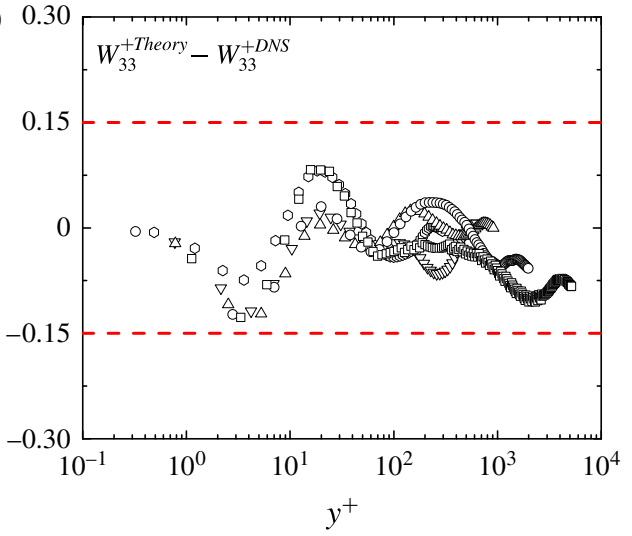

(b)

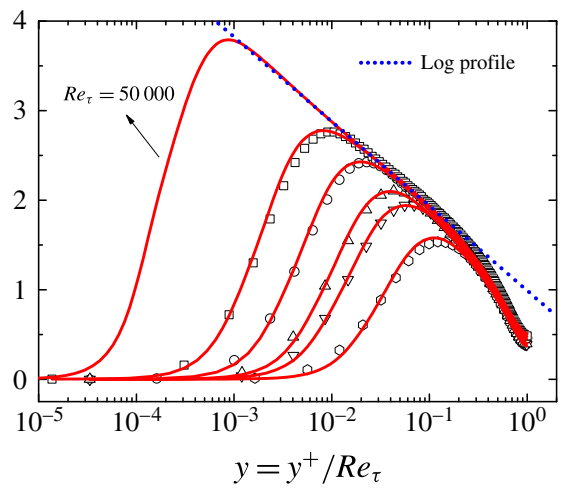

(d)

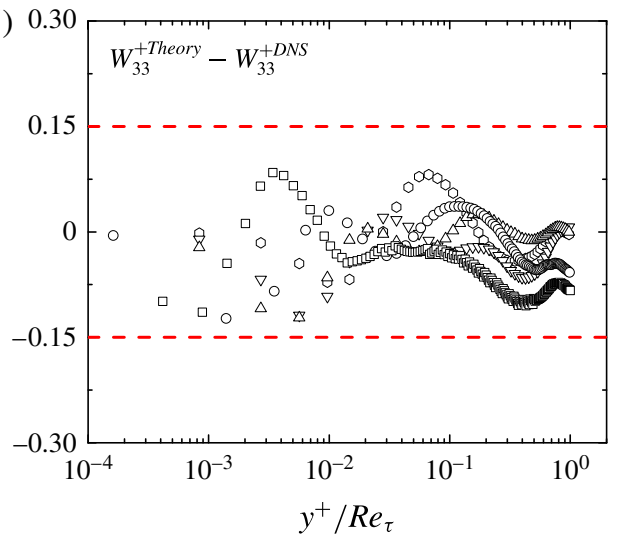

FIGURE 8. (Colour online) Validation of (5.3) - solid lines - for spanwise Reynolds stress profiles compared with data. Also included is the prediction of $W_{33}^{+}$at $R_{\tau}=50000$. Note the log profile (1.4) - dotted line in $(b)$ - agreeing well with data and with (5.3); its slope $A_{33} \approx 0.41$ and the additive constant $B_{33} \approx 1.00$ are theoretically determined in (5.16). The departure between data and our theory (i.e. $W_{33}^{+ \text {Theory }}-W_{33}^{+D N S}$ ) is shown in $(c, d)$, which is bounded within \pm 0.15 . For legend, see figure 2 .

which are very close to the empirical fitting values $A_{33}=0.387$ and $B_{33} \approx 1.08$ by Lee $\&$ Moser (2015). Thus, from (5.6) we obtain a (approximate) log law, hence presenting an alternative derivation of Townsend's log law.

Figure 8 shows the resulting $W_{33}^{+}$profiles by (5.2) - using (5.9) - and (5.3), in good agreement with data. The departure is bounded within \pm 0.15 . To emphasize, for all the predictions in figures 7 and 8, quite surprisingly, only two new parameters are introduced for $\ell_{33}$, i.e. $\gamma=0.07$ in (5.5) (or the peak location $y_{33 p}^{+} \approx 46$ ) and $\kappa_{33}^{\prime}=0.43$ in (5.8), both fixed for all $R e$. To our knowledge, this is the first time an analytical $W_{33}^{+}$profile for the entire flow domain has been obtained. Moreover, the $\log$ profile (1.4) with its slope and additive constant determined in (5.16) is also plotted in figure $8(b)$, which agrees not only with DNS data, but also with (5.3). A prediction at $R e_{\tau}=50000$ is included in the figure, awaiting future measurements for validation.

\section{Streamwise Reynolds stress $W_{11}$}

The canonical formula for $\ell_{11}^{+}$in $(2.32)$ and hence $W_{11}^{+}$:

$$
W_{11}^{+}=W_{12}^{+} \ell_{11}^{+2} / \ell_{12}^{+2}
$$



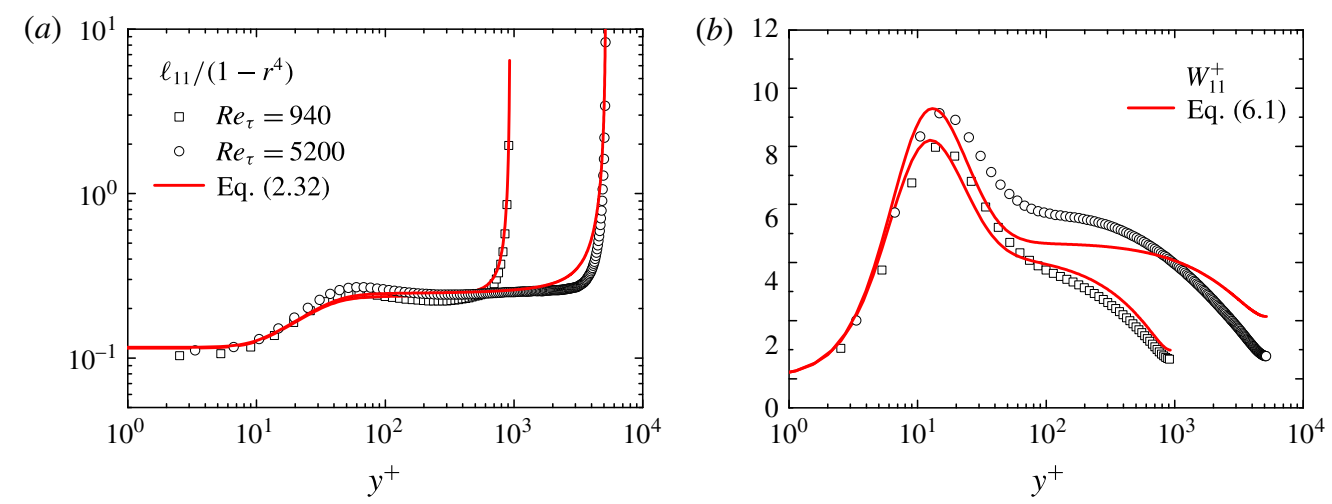

FIgURE 9. (Colour online) Canonical four-layer formula (2.32) (a) and the resulting $W_{11}^{+}$ profiles $(6.1)(b)$ compared with data, showing an underestimation in the bulk flow region at $R e_{\tau}=5200$.

are compared with data in figure 9 . While the inner peak is characterized well, $W_{11}^{+}$is underestimated in the bulk (particularly for high $R e$ ) where an modification is needed. Below we separately discuss the inner and outer regions.

\subsection{Inner profile of $W_{11}^{+}$}

As in the preceding sections, we let $c_{11}=c_{11}^{\prime} / \sqrt{1+r_{\text {core }}^{2}}$ in (2.32) so that $\ell_{11}^{+} \approx c_{11}^{\prime} y^{+}$as $y^{+} \rightarrow 0$, and let $c_{11}^{\prime}=\kappa_{11} \sqrt{y_{\text {sub }}^{+} / y_{\text {buf }}^{+}}$so that $\ell_{11}^{+} \approx \kappa_{11} y^{+}$as $y^{+} \gg y_{\text {buf }}^{+}$. Since (3.4) shows that $\ell_{12}^{+} \approx\left(y^{+} / y_{\text {sub }}^{+}\right)^{3 / 2}$, we have $W_{12}^{+} \approx 1-2 /\left[1+\sqrt{1+4\left(y^{+} / y_{\text {sub }}^{+}\right)^{3}}\right]$ (where $y^{+} / \operatorname{Re}_{\tau}$ is small and ignored). Thus, $W_{11}^{+}$for the inner flow region is approximated as

$$
W_{11 \text { Inner }}^{+}=W_{12}^{+} \ell_{11}^{+2} / \ell_{12}^{+2} \approx \frac{22.3 \kappa_{11}^{2}}{y^{*}}\left[1-\frac{2}{1+\sqrt{1+4\left(y^{*}\right)^{3}}}\right],
$$

where $y_{\text {sub }}^{+3} / y_{\text {buf }}^{+} \approx 22.3$ and $y^{*} \equiv y^{+} / y_{\text {sub }}^{+}=y^{+} / 9.7$ have been substituted into (6.2). Solving $\mathrm{d} W_{11}^{+} / \mathrm{d} y^{*}=0$ using (6.2), the inner peak location $y_{c}^{*}$ is then obtained:

$$
y_{c}^{*} \approx \sqrt[3]{2}
$$

and hence $y_{11 p i}^{+}=\sqrt[3]{2} y_{\text {sub }}^{+}$(scaled in viscous units). This estimation of $y_{11 p i}^{+}$, coinciding with the location of maximum production in (3.5), is independent of $\kappa_{11}$ and close to the value $y^{+} \approx 14$ observed from data (Pope 2000; Lee \& Moser 2015).

Moreover, the inner peak value is found to be determined by $\kappa_{11}$ by substituting (6.3) into (6.2), i.e.

$$
W_{11 p i}^{+} \approx 8.8 \kappa_{11}^{2}, \quad \text { or } \quad \kappa_{11}=\sqrt{W_{11 p i}^{+} / 8.8}
$$

Thus, $\kappa_{11}$ can be obtained from the measured inner peak value of $W_{11 p i}^{+}$. Figure 10(a) shows the measured $\kappa_{11}$ based on the DNS data and the Princeton pipe data (Hultmark et al. 2012). It seems that $\kappa_{11}$ saturates to a constant value around 1.02 (due to 
(a)

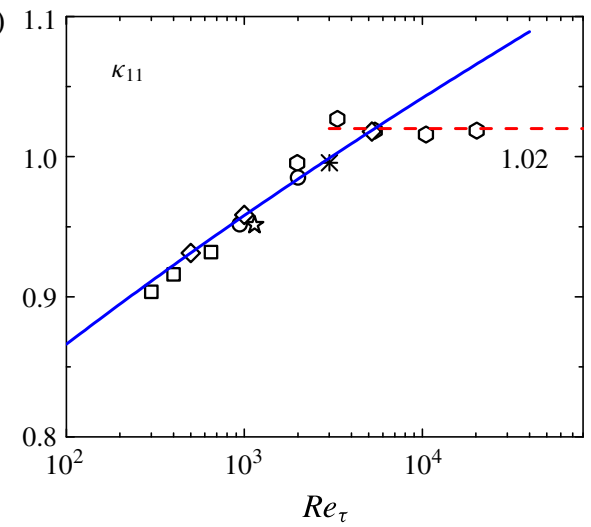

(b)

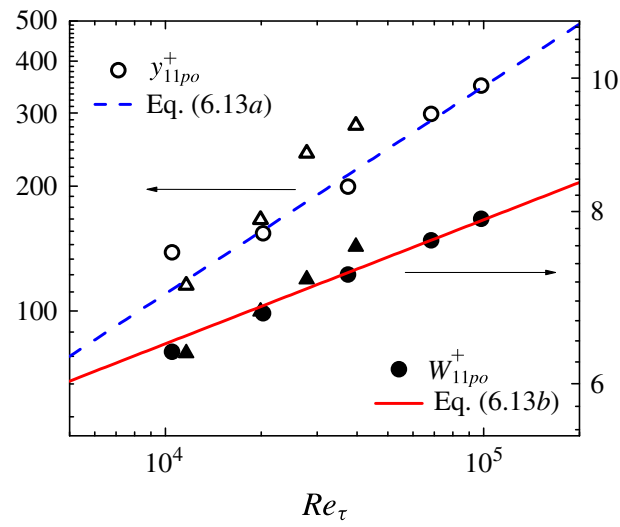

FIgURE 10. (Colour online) (a) Measurement of $\kappa_{11}$ by the inner peak value $W_{11 p i}^{+}$using (6.4). Dashed line denotes $\kappa_{11}=1.02$ resulting from $W_{11 p i}^{+}=9.2$ for Princeton pipe; solid line results from the empirical observation $W_{11 p i}^{+}=3.66+0.642 \ln \operatorname{Re}_{\tau}$ (Lee \& Moser 2015). Data: hexagon - Princeton pipe (Hultmark et al. 2012); other symbols are the same as in figure 3 for DNS data. (b) Predictions of outer peak location $y_{11 p o}^{+}$(left axis) using (6.13a) - dashed line - and of peak value $W_{11 p o}^{+}$(right axis) using $(6.13 b)$ - solid line; circles are Princeton pipe data and triangles are CICLoPE data; open $-y_{11 p o}^{+}$, solid $-W_{11 p o}^{+}$.

$W_{11 p i}^{+} \approx 9.2$ ) for $R e_{\tau} \gtrsim 5000$ of the Princeton pipe. However, if $W_{11 p i}^{+}$continues to grow with increasing $R e$, as that observed in TBL (Degraaff \& Eaton 2000; Metzger et al. 2001; Hutchins et al. 2009; Sillero et al. 2013), so also will $\kappa_{11}-$ according to (6.4). Issues related to this saturation are further discussed below.

Note that current channel flow DNS data show a logarithmic growth of $W_{11 p i}^{+}$as $R e_{\tau}$ increases (Lozano-Duran \& Jimenez 2014; Lee \& Moser 2015), which was also observed in TBL (Klewicki \& Falco 1990; Degraaff \& Eaton 2000; Metzger et al. 2001; Hutchins et al. 2009; Sillero et al. 2013; Marusic et al. 2015). Using the empirical formula $W_{11 p i}^{+}=3.63+0.65 \ln R e_{\tau}$ by Lozano-Duran \& Jimenez (2014) or $3.66+0.642 \ln R e_{\tau}$ by Lee \& Moser (2015), the resultant $\kappa_{11}$ from (6.4) is indicated by the solid line in figure $10(a)$, agreeing well with data at moderate $R e$. However, for $R e_{\tau}>5000$, Princeton pipe data (Hultmark et al. 2012) shows an invariant inner peak $W_{11 p i}^{+} \approx 9.2$, and hence the saturated $\kappa_{11} \approx 1.02$. This saturation is in contrast to Willert et al. (2017), where they report that the inner peak $W_{11 p i}^{+}$keeps increasing in their $R e$ range for $R e_{\tau}$ from 5000 to 40000 , and $W_{11 p i}^{+} \approx 9.8$ at $R e_{\tau}=40000$. The growth of the inner peak at finite $R e_{\tau}$ (up to 20000) is attributed to the inner-outer interaction; see Marusic, Baars \& Hutchins (2017) for more details.

Here, to match Princeton pipe data (Hultmark et al. 2012), $\kappa_{11}=1.02$ is adopted for $R e_{\tau}>5000$. As shown in figure 11(a), the inner profile given by (6.2) agrees well with high-Re pipe data, where $\kappa_{11}=1.02$ is the only new parameter. Moreover, figure $11(b)$ shows the similar comparison for the CICLoPE data, where $\kappa_{11}=1.04$ is adopted for the current $R e$ values (the inner profiles for $R e_{\tau}$ above 20000 are not shown because of data uncertainty on the peak value). Note that in figure $11(b), y^{*}=y^{+} / 12$ (instead of $y^{*}=y^{+} / 9.7$ ) is redefined in (6.2) - to match a larger $y_{11 p i}^{+} \approx 15-16$ of the CICLoPE data. One can check that the inner peak is still located at (6.3), and hence the invariance of (6.4); but the new definition of $y^{*}$ now leads to $y_{11 p i}^{+}=12 y_{c}^{*} \approx 15$, hence matching the CICLoPE data. A slight difference between Princeton pipe and CICLoPE data is also found in the outer flow region, which is addressed below. 
(a)

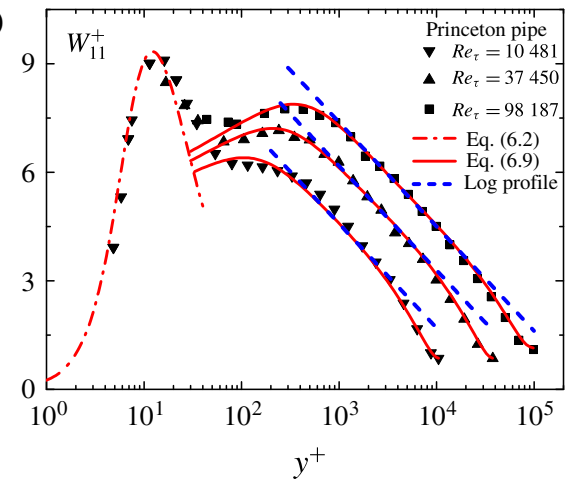

(b)

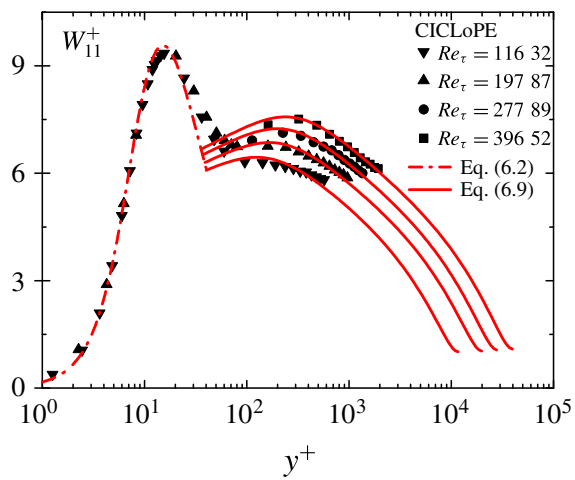

FIGURE 11. (Colour online) Inner profile by (6.2) - dash-dotted line - and the outer profile by (6.9) - solid line - compared with high $R e$ data in (a) Princeton pipe (Hultmark et al. 2012) and (b) CICLoPE (Willert et al. 2017). Note that $y^{*}$ in (6.2), and $c$ and $\gamma_{m}$ in (6.9) are slightly different between Princeton and CICLoPE datasets. Also included in (a) are $\log$ profiles of (1.3) - dashed lines - with $A_{11}=1.25$ and $B_{11}=1.63$ theoretically determined in (6.17).

\subsection{Outer profile with the mesolayer modification}

Note that according to the canonical four-layer descriptions (2.32) and (2.31), $W_{11}^{+}$ should exhibit a constant plateau in the log layer, namely

$$
W_{11}^{+}(r)=W_{12}^{+}\left(\ell_{11}^{+} / \ell_{12}^{+}\right)^{2} \approx \kappa_{11}^{2} / \kappa^{2} .
$$

However, this contrasts the recently observed outer peak (Hultmark et al. 2012; Willert et al. 2017). To characterize this peak, a mesolayer model was introduced by us (Chen et al. 2015) based on an anomalous scaling consideration, which further leads to a notable improvement of the $k-\omega$ model (Wilcox 2006) in predicting the entire $W_{11}^{+}$profile (Chen, Hussain \& She 2016a). Note that the mesolayer concept has been proposed before (Afzal 1982; Wosnik et al. 2000; Hultmark 2012), and we define it as the region above the buffer layer and ending at the peak location of the Reynolds shear stress (i.e. $y_{12 p}^{+}$in (3.9)).

Our definition of mesolayer yields an phenomenological explanation of the critical $\operatorname{Re}$ (i.e. $\operatorname{Re}_{\tau}^{c} \approx 10^{4}$ ) above which the outer peak emerges. Note that $y_{12 p}^{+}$was defined as the location of the 'critical layer' by Sreenivasan (1988), and as the demarcation of the inner-outer region by Klewicki et al. (2012). Accordingly, we consider $y_{12 p}^{+}$ $\left(=\sqrt{R e_{\tau} / \kappa}\right)$ as a typical height of near-wall coherent structures (or wall-attached eddies). As $R e$ increases, $y_{12 p}^{+}$will increase, indicating that such attached eddies move away from wall and finally enter the quasi-balance region (balance between production and dissipation of total kinetic energy) - the near-wall boundary is $y_{Q}^{+} \approx 150$ according to DNS data (Del Alamo \& Jimenez 2006). Hence, $y_{12 p}^{+}=y_{Q}^{+}$yields a critical $R e_{\tau}=\kappa y_{Q}^{+2} \approx 10100$. For small $R e$, these eddies are outside the quasi-balance zone; until $R e_{\tau}>10100$, they fully enter the quasi-balance zone, which may suppress local dissipation and result in the outer peak of $W_{11}^{+}-$in analogy to the coherent vortex filaments introducing scaling anomaly in the cascade of isotropic turbulence (She \& Leveque 1994). It is still a speculation, which may be complementary to the effect of LSM or VLSM (Hultmark et al. 2013; Vincenti et al. 2013; Vallikivi et al. 2015). 
Also, the non-equilibrium feature of dissipation (Vassilicos 2015) in the region of the outer peak is an interesting issue to pursue, as the non-local energy flux from the near-wall production peak may be important. A study of the two-point statistics (such as the velocity structure function) would provide evidence of the complex interplay between the non-local spatial and spectral energy fluxes, which requires more studies involving both the Reynolds stress equations and the spectral transfer equations.

Based on the mesolayer concept, Chen et al. (2015) postulate an anomalous scaling model for the ratio between $\ell_{11}$ and $\ell_{12}$, i.e.

$$
\theta_{\text {meso }}=c\left(y^{+}\right)^{\gamma_{b}}\left[1+\left(y^{+} / y_{\text {meso }}^{+}\right)^{2}\right]^{\left(\gamma_{m}-\gamma_{b}\right) / 2},
$$

where $y_{\text {meso }}^{+}=y_{12 p}^{+}=\sqrt{R e_{\tau} / \kappa}$. It readily leads to the outer profile of $W_{11}^{+}$:

$$
W_{11 \text { meso }}^{+}=W_{12}^{+} \theta_{\text {meso }}^{2}=c^{2} r\left(y^{+}\right)^{2 \gamma_{b}}\left[1+\left(y^{+} / y_{\text {meso }}^{+}\right)^{2}\right]^{\gamma_{m}-\gamma_{b}} .
$$

Compared to models for the outer $W_{11}^{+}$profile by Vassilicos et al. (2015) and Laval et al. (2017) (with eight parameters), equation (6.7) agrees equally well with data using much fewer parameters (only three). These three are: (1) the scaling exponent $\gamma_{b}=0.05$ quantifying the growth of $W_{11}^{+}$before reaching its outer peak; (2) the second exponent $\gamma_{m}=-0.09$ quantifying the decay of $W_{11}^{+}$after its outer peak; and (3) the global proportionality coefficient $c$ determining the magnitude of outer profile. The two exponents are believed to be related to intermittent cascade dynamics in the bulk developed (more isotropic) turbulence. Presently, they are measured from experimental data (Chen et al. 2015), which seem to be invariant with $R e$ - to be further elucidated in the future.

On the other hand, the value of $c$ for each $R e$ is determined by the following procedure. Note that (6.7) implies $W_{11 c}^{+}=0$ at the centreline $r=0$, due to a lack of the centre core layer factor in (6.6), which can be readily rectified by incorporating the core layer asymptote $\ell_{11} / \ell_{12} \propto\left(1+\left(r_{\text {core }} / r\right)^{2}\right)^{1 / 4}$ as $r \rightarrow 0$ from (2.34). This yields

$$
\theta_{\text {outer }}=c\left(y^{+}\right)^{\gamma_{b}}\left(1+\left(\frac{y^{+}}{y_{\text {meso }}^{+}}\right)^{2}\right)^{\left(\gamma_{m}-\gamma_{b}\right) / 2} \frac{\left(1+\left(r_{\text {core }} / r\right)^{2}\right)^{1 / 4}}{\left(1+r_{\text {core }}^{2}\right)^{1 / 4}},
$$

where the factor $\left(1+r_{\text {core }}^{2}\right)^{1 / 4}$ guarantees that $\theta_{\text {outer }} \rightarrow \theta_{\text {meso }}$ as $r \rightarrow 1$. Therefore, the expression for the entire outer profile of $W_{11}^{+}$is

$$
W_{11 \text { Outer }}^{+}=W_{12}^{+} \theta_{\text {outer }}^{2}=c^{2}\left(y^{+}\right)^{2 \gamma_{b}}\left(1+\left(\frac{y^{+}}{y_{\text {meso }}^{+}}\right)^{2}\right)^{\gamma_{m}-\gamma_{b}} \frac{\sqrt{r^{2}+r_{\text {core }}^{2}}}{\sqrt{1+r_{\text {core }}^{2}}} .
$$

One can check as $r \rightarrow 1, W_{11 \text { Outer }}^{+} \rightarrow W_{11 \text { meso }}^{+}$(hence (6.9) $\rightarrow$ (6.7)). Moreover, at $r=0$ (i.e. $y^{+}=R e_{\tau}$ ), we obtain a non-zero centreline value of $W_{11}^{+}$from (6.9):

$$
W_{11 c}^{+}=c^{2} R e_{\tau}^{2 \gamma_{m}} y_{12 p}^{+2\left(\gamma_{b}-\gamma_{m}\right)} \frac{r_{\text {core }}}{\sqrt{1+r_{\text {core }}^{2}}} .
$$

Now, similar to (5.6), we invoke Townsends' $R e$-similarity hypothesis, namely, $W_{c 11}^{+}$is expected to be independent of $R e$, which yields $c \propto R e_{\tau}^{-\left(\gamma_{b}+\gamma_{m}\right) / 2}$. Denote $c=c^{\prime} R e_{\tau}^{-\left(\gamma_{b}+\gamma_{m}\right) / 2}$ and substitute this $c$ and $y_{12 p}^{+}$into (6.10), yielding

$$
c^{\prime}=W_{c 11}^{+1 / 2}\left(1+r_{\text {core }}^{2}\right)^{1 / 4} \kappa^{\gamma_{b}-\gamma_{m}} / \sqrt{r_{\text {core }}} .
$$


Using $r_{\text {core }}=0.27, \kappa=0.45$, and the centreline measurement $W_{11 c}^{+} \approx 0.86$ from the EXP pipe at $R e_{\tau}=10481$ (Hultmark et al. 2012), we obtain $c^{\prime} \approx 1.72$, which yields

$$
c=c^{\prime} \operatorname{Re}_{\tau}^{-\left(\gamma_{b}+\gamma_{m}\right) / 2} \approx 1.72 R e_{\tau}^{0.02}
$$

Figure 11(a) shows that (6.9) agrees well with the high-Re data of the Princeton pipe. Recall the model of Vassilicos et al. (2015) and Laval et al. (2017) for the outer profile of $W_{11}^{+}$; it is built on a match of two piecewise functions which are obtained by the integration of a (presumed) four-layer power-law spectrum (including a $k_{x}^{-1}$ spectrum corresponding to the logarithmic profile by Townsend). However, it is difficult for such a spectral model to locate precisely the log region (particularly for small $R e$ ). In contrast, equation (6.9) describes the simple multilayer structure, needing no spectral description, and involving far fewer parameters. Figure 11 shows that (6.9) characterizes well both the smooth variation of the data across the outer peak and the gradual deviation of the data from the logarithmic distribution near the centreline, which were not described yet in Laval et al. (2017).

The comparison between (6.9) and the revised CICLoPE data is shown in figure $11(b)$. Note that only a small part of $W_{11}^{+}$is measured in the outer flow region of CICLoPE, which differs from the Princeton pipe data slightly. In particular, beyond the outer peak, $W_{11}^{+}$declines more slowly in CICLoPE, hence suggesting a larger $\gamma_{m}=-0.07$ (compared to -0.09 ) for the four profiles shown in figure $11(b)$. Moreover, for $R e_{\tau}=27789$ and 39652, the outer peak value of CICLoPE is slightly larger than the Princeton pipe at similar values of $\operatorname{Re}_{\tau}$, thus implying a larger magnitude parameter $c$ for these two profiles (i.e. $c=1.75 R e_{\tau}^{0.02}$ ). Except for these two changes, all the other parameters in (6.9) stay the same as for the Princeton pipe, and the resulting profiles agree closely with the CICLoPE data. As a reminder, according to the Princeton data in figure $11(a), \gamma_{m}=-0.09$ and $c=1.72 R e_{\tau}^{0.02}$ match well with data at even higher $\operatorname{Re}$ (i.e. $\operatorname{Re}_{\tau}=37450$ and 98187 ). It is thus unknown whether the small changes of $c$ and $\gamma_{m}$ are physically necessary, or simply due to data uncertainty (an issue that has been emphasized for CICLoPE by Willert et al. (2017)).

\subsection{Scaling of the $W_{11}^{+}$outer peak}

Now, using the three parameters $\left(\gamma_{b}=0.05, \gamma_{m}=-0.09\right.$ and $\left.c^{\prime}=1.72\right)$, let us predict the $R e$-scaling of the outer peak value $W_{11 p o}^{+}$and its $y$-location $y_{11 p o}^{+}$. Solving $\partial_{y^{+}} W_{11}^{+}=$ 0 using (6.9), which actually is $\partial_{y^{+}} \theta_{\text {meso }} \approx 0$ since $r \approx 1$, one has the outer peak location:

$$
y_{11 p o}^{+}=\sqrt{-\gamma_{b} / \gamma_{m}} y_{\text {meso }}^{+} \approx 1.1 R e_{\tau}^{1 / 2} .
$$

Moreover, substituting (6.13a), $y_{\text {meso }}^{+}=\sqrt{R e_{\tau} / \kappa}$ and (6.12) into (6.7) yields the outer peak value:

$$
W_{11 p o}^{+}=c^{\prime 2}\left(-\gamma_{b} / \gamma_{m}\right)^{\gamma_{b}}\left(1-\gamma_{b} / \gamma_{m}\right)^{\gamma_{m}-\gamma_{b}} \operatorname{Re}_{\tau}^{-\gamma_{m}} \approx 2.8 R \boldsymbol{R}_{\tau}^{0.09}
$$

Predictions by $(6.13 a)$ and (6.13b) compared with data are shown in figure $10(b)$. The agreement is quite good, though the triangles (CICLoPE data) at the two largest $R e$ are a little higher than the circles (Princeton pipe data) - due to the aforementioned larger $\gamma_{m}$ and $c$ for CICLoPE.

To emphasize, $y_{11 p o}^{+} \propto R e_{\tau}^{1 / 2}$ in (6.13a) is obtained by the scaling of $y_{\text {meso }}^{+}$, and this scaling is independent of the values of $c^{\prime}, \gamma_{b}$ and $\gamma_{m}$. This is self-consistent with 
the preceding suggested scenario that $y_{\text {meso }}^{+}$(i.e. typical height of near-wall coherent structures) entering the quasi-balance zone causes the anomalous dissipation, and hence the outer peak. This $R e_{\tau}^{1 / 2}$ scaling is also different from the $R e_{\tau}^{2 / 3}$ scaling by McKeon \& Sharma (2010), where they argue that the outer peak corresponds to a specific mode whose propagation velocity equals the local mean velocity at the position where $U^{+}=2 U_{c}^{+} / 3$. Currently, both scalings agree with data (within data uncertainty) in the present $R e$ range, while further higher-Re data are needed to indicate the preferred of the two scalings (note that $R e_{\tau}^{1 / 2}$ scaling is better for TBL data of Mathis, Hutchins \& Marusic (2009)).

According to $(6.13 b)$, the outer peak will exceed the inner peak - taking the aforementioned constant value $9.2-$ at $R e_{\tau}=(9.2 / 2.8)^{1 / 0.09} \approx 5.5 \times 10^{5}$. (However, if the inner peak continues to increase, this $R_{\tau}$ will be larger.) Prediction of this specific $R e_{\tau}$, approximately six times larger than the maximum $R e_{\tau}=98187$ in the Princeton pipe data (Hultmark et al. 2012), awaits verification when data become available.

\subsection{Townsend's log profile for $W_{11}^{+}$}

Another important issue concerns the theoretical explanation of the recently observed logarithmic distribution (Hultmark et al. 2012), i.e. $W_{11}^{+}=-1.25 \ln y+1.61$. As introduced before, previous theories of the log profile build on the $k_{x}^{-1}$ spectrum (Townsend 1976; Perry et al. 1986), which is, however, not fully supported by experimental pipe data (Rosenberg et al. 2013). Alternatively, Hultmark (2012) suggested investigating the diagnostic function $\Gamma_{11}=-y d_{y} W_{11}^{+}$to check the log profile. Here, similar to (5.12), we can calculate $\Gamma_{11}$ from (6.9), and hence theoretically estimate the values of the log-law slope $A_{11}$ and the additive constant $B_{11}$, presented below.

Since the log profile region is beyond the outer peak $\left(y^{+} \gg y_{\text {meso }}^{+}\right.$and $\left.r \approx 1\right)$, equation (6.9) is approximated as

$$
W_{11}^{+}=c^{\prime 2} \kappa^{\gamma_{m}-\gamma_{b}} r y^{2 \gamma_{m}}
$$

from which we have

$$
\Gamma_{11}=c^{\prime 2} \kappa^{\gamma_{m}-\gamma_{b}} y^{2 \gamma_{m}}\left[1-\left(1+2 \gamma_{m}\right) r\right]
$$

Again, the value of the approximate plateau of our theoretical $\Gamma_{11}$ can be represented by its minimum value $\Gamma_{11 \mathrm{~min}}$, which can be obtained by solving $\mathrm{d} \Gamma_{11} / \mathrm{d} y=0$ using (6.15). The results are

$$
\Gamma_{11 \text { min }}=c^{\prime 2} \kappa^{\gamma_{m}-\gamma_{b}}\left(\frac{2 \gamma_{m}}{1+2 \gamma_{m}}\right)^{1+4 \gamma_{m}} \approx 1.25
$$

which is located at

$$
y_{11 \Gamma}=\left(\frac{2 \gamma_{m}}{1+2 \gamma_{m}}\right)^{2} \approx 0.048 .
$$

Repeating the calculation for $B_{33}$ in (5.16), the logarithmic additive constant $B_{11}$ can be obtained by matching (6.14) with (1.3) at the specific location $y=y_{11 \Gamma}$. Substituting $y=y_{11 \Gamma} \approx 0.048$ into $(6.14)$ yields $W_{11 \Gamma}^{+} \approx 5.43$; therefore

$$
A_{11}=\Gamma_{11 \min } \approx 1.25, \quad B_{11}=W_{11 \Gamma}^{+}+A_{11} \ln y_{11 \Gamma} \approx 1.63
$$


which are very close to the empirical values of $A_{11}=1.25$ and $B_{11}=1.61$ obtained by Hultmark et al. (2012). Figure 11 shows our theoretical log law with parameters in (6.17), in good agreement with both (6.9) and data. Thus, (6.9) leads to a local approximate log law, hence presenting an alternative derivation of the Townsend's log profile.

\subsection{Composite expression for the entire flow}

Now, we incorporate $\theta_{\text {outer }}$ (or $\theta_{\text {meso }}$ ) with $\alpha_{11}$ (and hence $\ell_{11}$ ) to form a composite expression for the entire flow, without introducing any additional parameter. Since $\alpha_{11} \propto 1 / \sqrt{y^{+}}$as $y^{+} \rightarrow 0$, we now modify the $y^{+\gamma_{b}}$ factor in $\theta_{\text {outer }}$ to match the near-wall scaling $1 / \sqrt{y^{+}}$and obtain the following improved $\alpha_{11}$ (explained below):

$$
\alpha_{11}^{\prime}=\frac{c_{1} y_{\text {buf }}^{+1 / 2}}{\sqrt{y^{+}}}\left(1+\left(\frac{y^{+}}{y_{B}^{+}}\right)^{4}\right)^{\left(1 / 2+\gamma_{b}\right) / 4}\left(1+\left(\frac{y^{+}}{y_{\text {meso }}^{+}}\right)^{2}\right)^{\left(\gamma_{m}-\gamma_{b}\right) / 2}\left(1+\left(\frac{r_{\text {core }}}{r}\right)^{2}\right)^{1 / 4}
$$

and hence

$$
\ell_{11}^{\prime}=\ell_{12}^{+} \alpha_{11}^{\prime}, \quad \text { and } \quad W_{11}^{+}=W_{12}^{+}\left(\ell_{11}^{\prime} / \ell_{12}\right)^{2}=W_{12}^{+}\left(\alpha_{11}^{\prime}\right)^{2} .
$$

It can be easily checked that $\alpha_{11}^{\prime}=\alpha_{11}$ for $y^{+} \ll y_{B}^{+}$, and $\alpha_{11}^{\prime}=\theta_{\text {outer }}$ for $y^{+} \gg y_{B}^{+}$; note that the second condition implies $c_{1} y_{b u f}^{+1 / 2} / y_{B}^{+\left(1 / 2+\gamma_{b}\right)}=c /\left(1+r_{\text {core }}^{2}\right)^{1 / 4}$, which leads to the definition of $y_{B}^{+}$:

$$
y_{B}^{+}=\left[c_{1} y_{\text {buf }}^{+1 / 2}\left(1+r_{\text {core }}^{2}\right)^{1 / 4} / c\right]^{2 /\left(1+2 \gamma_{b}\right)} .
$$

Substituting the global coefficients $c_{1}=c_{11} /\left(c_{12} y_{\text {buf }}^{+1 / 2}\right), c_{11}=\kappa_{11} y_{\text {sub }}^{+1 / 2} /\left[y_{\text {buf }}^{+}\left(1+r_{\text {core }}^{2}\right)\right]^{1 / 2}$, $c_{12}=\kappa y_{\text {sub }}^{+1 / 2} /\left[y_{\text {buf }}^{+}\left(1+r_{\text {core }}^{2}\right)^{1 / 4}\right]$ and $c=c^{\prime} R e_{\tau}^{-\left(\gamma_{m}+\gamma_{b}\right) / 2}$ into (6.20a) yields

$$
y_{B}^{+}=\left[\kappa_{11} y_{b u f}^{+1 / 2} /\left(c^{\prime} \kappa\right)\right]^{2 /\left(1+2 \gamma_{b}\right)} R e_{\tau}^{\left(\gamma_{b}+\gamma_{m}\right) /\left(1+2 \gamma_{b}\right)} \approx 48 R e_{\tau}^{-0.0364} .
$$

Here, the values of $\kappa=0.45, y_{b u f}^{+}=41, \kappa_{11}=1.02, c^{\prime}=1.72, \gamma_{b}=0.05$ and $\gamma_{m}=-0.09$ are used.

Substituting (6.20b) into (6.18) and hence (6.19), the resulting $W_{11}^{+}$profiles are shown in figure 12(a), in good agreement with data for the entire flow domain with a departure less than 1. Predictions for two larger $R e$ values at $R e_{\tau}=3 \times 10^{5}$ and $10^{6}$ are also plotted in the figure, awaiting future measurement for validation. The diagnostic function $\Gamma_{11}$ for the entire flow domain is also calculated from data and from (6.19), as shown in figure 12(b); the plateau value of the data in the bulk flow is well represented by $\Gamma_{\min }=1.25$ in $(6.16 a)$. That is why the $\log$ profile with $A_{11}=1.25$ and $B_{11}=1.63$ agrees well with the data in figure $12(a)$.

It is interesting to observe that for the two larger $R e$ values $\left(R e_{\tau}=3 \times 10^{5}\right.$ and $\left.10^{6}\right)$, a more extended logarithmic scaling is predicted by (6.18), and hence (6.19). This is in sharp contrast to the power-law formula by Barenblatt (1993) and Barenblatt \& Chorin (2004), where their power-law asymptotes to a $\log$ law due to a decreasing scaling exponent with increasing $R e$. Here, (6.18) predicts the (approximate) log profile for $W_{11}^{+}$based on three $R e$-invariant parameters, i.e. $c^{\prime}=1.72, \gamma_{b}=0.05$ and $\gamma_{m}=-0.09$. Moreover, equation (6.18) extends the log law to the centreline and to 
(a)

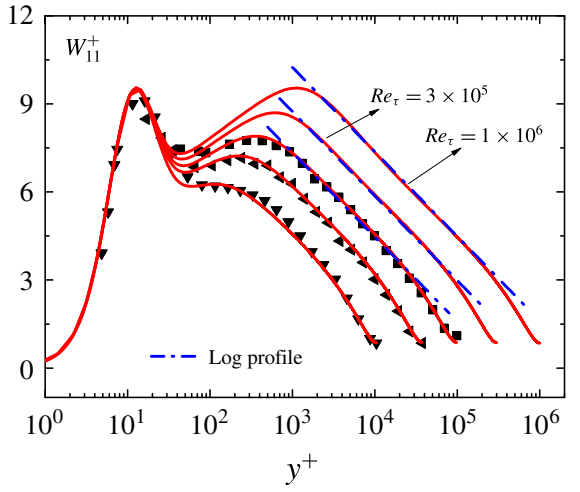

(b)

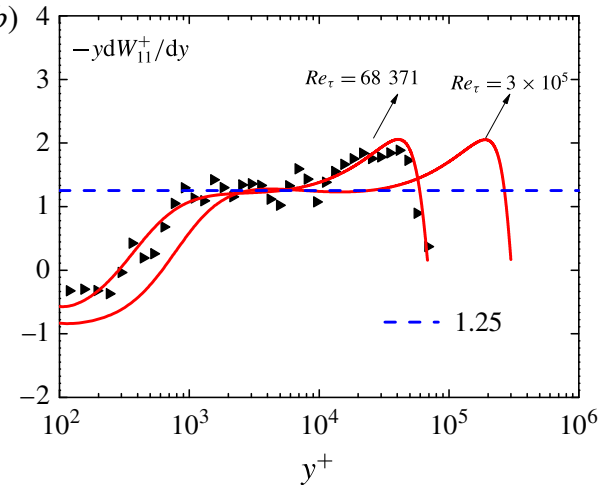

FIGURE 12. (Colour online) (a) EXP pipe data (Hultmark et al. 2012) denoted by symbols compared with (6.19) denoted by solid lines for $R e_{\tau}=10481$ (downward triangle), 37450 (leftward triangle) and 98187 (square) - showing good agreement. Our log profile $W_{11}^{+}=-1.25 \ln \left(y^{+} / R e_{\tau}\right)+1.63$ from (6.17) is marked in dash-dotted line. Also included are predictions for $R e_{\tau}=3 \times 10^{5}$ and $10^{6}$, awaiting future data for validation. $(b)$ The logarithmic diagnostic function $\Gamma_{11}=-y d_{y} W_{11}^{+}$for data (symbol) and using (6.19) (solid line) showing good agreement in the entire flow domain. The dashed line marks the $\log$ profile slope -1.25 given in (6.17); also included is the prediction of $\Gamma_{11}$ at $R e_{\tau}=3 \times 10^{5}$.

the wall, considerably beyond the previous scaling theories valid for a limited flow domain, hence showing the superiority of (6.18).

For small $R e$ values, using $\kappa_{11}$ in figure $10(a)$ and $y_{B}^{+}$in $(6.20 b)$, the resulting $W_{11}^{+}$ profiles by (6.19) are shown in figure 13. Note that in this figure, $\operatorname{Re}_{\tau}$ covers over two decades from 650 to 98187 . Compared to the canonical descriptions (figure $9 b$ ), the modified $W_{11}^{+}$profiles agree much better with data (figure $13 a$ ), although there is a slight mismatch in the buffer layer for small $R e$ (figure $13 c$ ). The agreement can be improved by slightly varying $\gamma_{m}$ (from -0.09 to -0.07 ). However, to limit the scope and the size of the paper, a detailed discussion of the finite Re-dependence of the parameters is not considered. Still, the improvement indicates that the anomalous scaling modification, although inspired from large- $R e$ data, also applies to moderate $R e$. The underpinning physics for the successful modifications of small-Re profiles need further studies.

Remember that $(6.13 b)$ predicts that the outer peak will exceed 9.2 (the presumed saturated inner peak value) at $R e_{\tau} \approx 5.5 \times 10^{5}$. According to $(6.20 b), y_{B}^{+}$decreases from approximately 41 to 31 for $R e_{\tau}$ varying from $10^{2}$ to $10^{5}$, and $y_{B}^{+} \approx 29$ at $R e_{\tau}=$ $5.5 \times 10^{5}$. For $R e \rightarrow \infty$, equation $(6.20 b)$ indicates that $y_{B}^{+} \rightarrow 0$, which seems to be questionable since $y_{B}^{+}$should saturate to a non-zero value larger than $y_{\text {sub }}^{+}=9.7$ (i.e. above the sublayer thickness). In fact, a non-zero $y_{B}^{+}$as $\operatorname{Re}_{\tau} \rightarrow \infty$ indicates that $\gamma_{b}+\gamma_{m} \rightarrow 0$. However, $y_{B}^{+}$decays very slowly with $R e$, and it is not until $R e_{\tau}>10^{20}$ that $y_{B}^{+}$becomes smaller than $y_{s u b}^{+}$- extremely far away from today's experiments and simulations. Thus, to re-emphasize, equation $(6.20 b)$ is quite robust for finite $R e$.

\section{Summary}

The symmetry approach developed in Part 1 (She et al. 2017) for the mean velocity profiles is extended here to quantify the (second-order) Reynolds stress tensor in channel and pipe flows. The canonical four-layer description of the Reynolds shear 
(a)

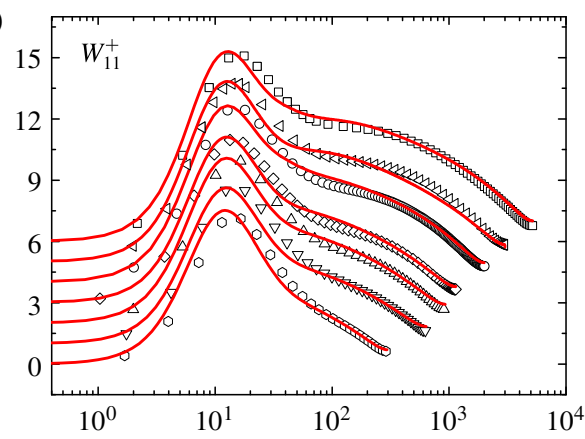

(c)

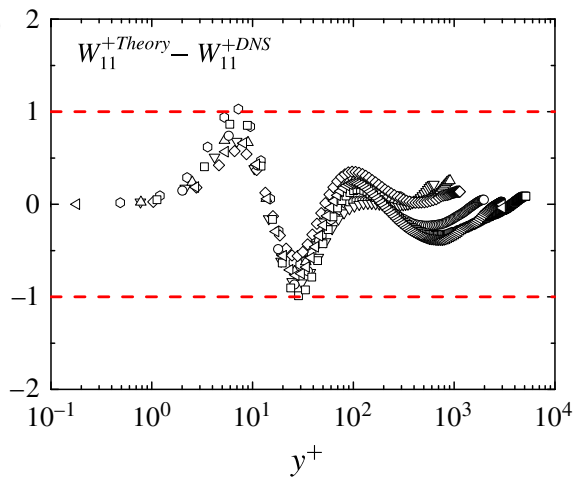

(b)

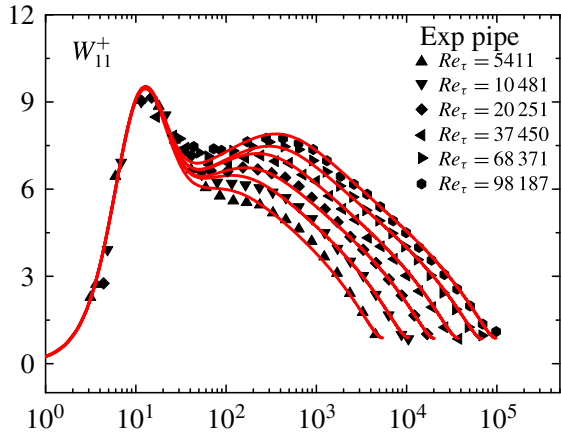

(d)

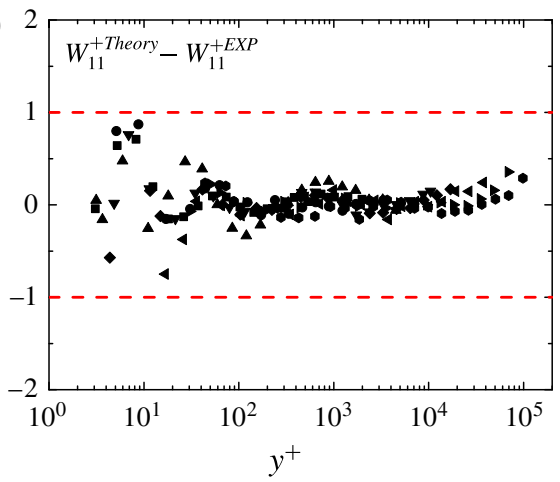

FIGURE 13. (Colour online) $W_{11}^{+}$obtained by anomalous scaling modification compared with data of (a) DNS (each profile has been vertically shifted by one unit for better display) and (b) Princeton pipe experiment (EXP) (Hultmark et al. 2012). The departure between data and our theory is shown in (c) (for DNS) and (d) (for EXP) - all bounded within \pm 1 . In addition to the DNS channel data in figure 2 , DNS pipe data are also included in $(a, c)$ at $R e_{\tau}=1142$ by $\mathrm{Wu} \&$ Moin (2008) (diamonds) and at $R e_{\tau}=3008$ by Ahn et al. (2015) (leftward triangles).

stress length $\ell_{12}$ is extended to similarly obtain those of the three other lengths, $\ell_{11}$, $\ell_{22}$ and $\ell_{33}$. Among these length functions, the layer thicknesses are found to be the same (except $r_{22 \text { core }}$ is $10 \%$ larger than $r_{\text {core }}$ ), while the scaling exponents are different due to different wall and centreline asymptotes, owing to different symmetries under dilations. $W_{12}^{+}$and $W_{22}^{+}$(active motions) agree well with the canonical descriptions; for $W_{11}^{+}$and $W_{33}^{+}$(inactive motions), small anomalous modifications yield more refined results, representing the effect of symmetry breaking, whose dynamic mechanism will be an intriguing topic for further study.

Let us summarize all parameters describing the multilayer structure of wall turbulence in channel and pipe flows: three layer-thickness parameters characterizing the canonical four-layers structure $\left(y_{\text {sub }}^{+}=9.7, y_{\text {buf }}^{+}=41, r_{\text {core }}=0.27\right.$, with slight modification of the last one $-r_{22 \text { core }}=0.3$ for $\left.\ell_{22}\right)$; four proportionality coefficients $\left(\kappa=0.45\right.$ for $\ell_{12} ; \kappa_{11}=1.02$ (for high $R e$ ), $\kappa_{22}=0.52, \kappa_{33}^{\prime}=0.43$ for $\ell_{11}, \ell_{22}$ and $\ell_{33}$, respectively); three anomalous scaling exponents $\left(\gamma=0.07\right.$ for $\ell_{33}, \gamma_{b}=0.05$ and $\gamma_{m}=-0.09$ for $\ell_{11}$ ); and finally a coefficient $c^{\prime}=1.72$ related to the outer peak of $W_{11}^{+}$. These parameters completely quantify the multilayer structures of the mean velocity, Reynolds shear stress and three turbulence intensities (including their $R e$-scalings and $y$-scalings), and each of these parameters is directly and independently measured from data. It would be interesting to test whether they remain invariant at higher $R e$. 
Using the above parameters, we theoretically obtain following results, agreeing well with data:

(i) the maximum turbulent production is located at $y^{+} \approx 12$;

(ii) the location of peak value $W_{12}^{+}$has a scaling transition from $5.7 R e_{\tau}^{1 / 3}$ to $1.5 R e_{\tau}^{1 / 2}$ at $R e_{\tau} \approx 3000$, with the peak magnitude $1-W_{12 p}^{+}$having a scaling transition from $8.5 R e_{\tau}^{-2 / 3}$ to $3.0 R e_{\tau}^{-1 / 2}$

(iii) the peak value $W_{33 p}^{+} \approx 0.84 R e_{\tau}^{0.14}\left(1-48 / R e_{\tau}\right)$;

(iv) the outer peak of $W_{11}^{+}$emerges above $R e_{\tau} \approx 10^{4}$ with its location scaling as $1.1 R e_{\tau}^{1 / 2}$ and its magnitude scaling as $2.8 R e_{\tau}^{0.09}$

(v) alternative derivations of Townsend's log profiles for inactive motions in the bulk, viz., $\overline{u^{\prime} u^{\prime}} \approx-1.25 \ln y+1.63$ and $\overline{w^{\prime} w^{\prime}} \approx-0.41 \ln y+1.00$.

The above results obtained from the concise four-layers structure, albeit slight modifications for inactive motions, demonstrate that (Reynolds stress) lengths are the desired similarity variables for quantifying wall turbulence. The reason why these lengths are assumed to have local dilation invariants is that they are believed to characterize spatial scales of (statistical) eddies of wall turbulence. According to structural ensemble dynamics (SED) in Part 1, these lengths are examples to represent the ensemble property of the flows, which are constrained by the wall and obey the dilation invariance ansatze postulated in Part 1. In other words, within each layer, eddies' scales are related by the simple power law (following the first or the second ansatz); and across different layers, eddies' scales obey the generalized power law (following the third ansatz). The good agreement between data and the multilayer descriptions illustrates that different components of Reynolds stresses are indeed organized into a self-similar family. Note that in contrast to the wall-attached hypothesis by (Townsend 1976) (mainly for the log layer), the current work provides a framework to define a family of eddies in the entire flow domain. The eddies' scales characterized by the lengths enable the quantification of mean distributions (e.g. mean velocity, fluctuation intensities), where more refined descriptions (such as anomalous scaling modifications of $\ell_{11}$ and $\ell_{33}$ ) can be pursued further.

Finally, owing to the universal nature of wall dilation symmetry, the analysis is applicable to many other wall flows with different flow conditions, such as incompressible (Wu et al. 2013; Chen \& She 2016) and compressible TBL (She et al. 2010; Zhang et al. 2012; Wu et al. 2017), roughness effects (She et al. 2012), with and without mild pressure gradients, and turbulent Rayleigh-Bénard convection (to be communicated soon). The results have also been extended to improve turbulent engineering models (Chen et al. 2015, 2016a). Note that the random dilation analysis on (approximate) balance equations, which take different forms in different layers, also can be applied to study the scalings of high-order correlations.

\section{Acknowledgements}

The authors thank C. E. Willert for sharing the CICLoPE data with us. This work is initially supported by the MOST 973 project 2009CB724100 of China, and then by National Nature Science Foundation of China with nos. 11452002, 11221062.

\section{Appendix A. Matching procedure}

The matching procedure developed in Part 1 is summarized here. Suppose we have two different power laws for two adjacent layers, for example, $\ell^{(I)}=c_{I} y^{\gamma_{I}}$ (layer I) and 
$\ell^{(I I)}=c_{I I} y^{\gamma_{I I}}$ (layer II). Recall the third ansatz in Part 1 composed of the two invariants $I_{1}$ and $I_{2}$, i.e.

$$
I_{2}=\gamma_{I} I_{1}+c\left(I_{1}\right)^{n}
$$

where $\gamma_{I}, c$ and $n$ are all constants. Substituting the definitions $I_{1}=\ell / y^{\gamma_{I I}}$ and $I_{2}=$ $(\mathrm{d} \ell / \mathrm{d} y) / y^{\gamma_{I I}-1}$ obtained from the dilations in the layer II into (A 1), and integrating the subsequent equation with respect to $y$, we obtain the following general scaling transition:

$$
\ell=c_{I} y^{\gamma_{I}}\left(1+\left(y / y_{c}\right)^{p}\right)^{\left(\gamma_{I I}-\gamma_{I}\right) / p},
$$

where $y_{c}=\left(c_{I} / c_{I I}\right)^{1 /\left(\gamma_{I I}-\gamma_{I}\right)}$ is the cross $y$-location satisfying $\ell^{(I)}=\ell^{(I I)}$, and the transition sharpness $p=(1-n)\left(\gamma_{I I}-\gamma_{I}\right)$; note that $\ell$ represents any of $\ell_{11}, \ell_{12}, \ell_{22}$ and $\ell_{33}$. It can be checked that (A 2) smoothly connects the above two adjacent power laws together.

Now we use the above ansatz to obtain the multilayer formulae of the stress length functions. Let us take $\ell_{22}$ as an example to show how the composite formula is obtained (note that $\ell_{12}$ has been obtained in Part 1). First, we apply (A 1) and (A 2) for $\ell_{22}$ in the viscous sublayer and buffer layer. For this case, $\ell_{22}^{(I)}=I_{1}^{s u b} y^{+2}$ representing the power law for the sublayer, and $\ell_{22}^{(I I)}=I_{1}^{b u f} y^{+5 / 2}$ for the buffer layer. Substituting $\ell_{22}$ into (A 1) and carrying out the integration to (A 2) we obtain

$$
\ell_{22}^{+(s u b-b u f)}=I_{1}^{s u b} y^{+2}\left(1+\left(\frac{y^{+}}{y_{\text {sub }}^{+}}\right)^{p}\right)^{1 / 2 p}=c_{22}^{\prime} y^{+2}\left(1+\left(\frac{y^{+}}{y_{\text {sub }}^{+}}\right)^{4}\right)^{1 / 8}
$$

where $y_{\text {sub }}^{+}=\left(I_{1}^{\text {sub }} / I_{1}^{b u f}\right)^{2}$ is the cross $y^{+}$-location for the two layers, $c_{22}^{\prime}=I_{1}^{\text {sub }}$ is the proportionality coefficient, and the transition sharpness $p=4$ is the same as in Part 1 .

Then, for buffer and log layers, $\ell_{22}^{(I)}=I_{1}^{b u f} y^{+5 / 2}$ and $\ell_{22}^{(I I)}=I_{1}^{\log } y^{+}\left(=\kappa_{22} y^{+}\right)$. Repeating the above derivation procedure, we obtain the scaling transition from buffer to log layers:

$$
\ell_{22}^{+(b u f-l o g)}=I_{1}^{b u f} y^{+5 / 2}\left(1+\left(\frac{y^{+}}{y_{\text {buf }}^{+}}\right)^{4}\right)^{-3 / 8},
$$

where $y_{\text {buf }}^{+}=\left(I_{1}^{\log } / I_{1}^{\text {buf }}\right)^{2 / 3}$.

Further, using the following multiplicative rule (Van Dyke 1964), i.e.

$$
\phi^{I-I I I}=\phi^{I-I I} \phi^{I I-I I I} / \phi^{\text {Common }}
$$

which corresponds to

$$
\ell_{22}^{+ \text {Inner }}=\ell_{22}^{+(\text {sub-buf })} \ell_{22}^{+(\text {buf }-\log )} /\left(I_{1}^{\text {buf }} y^{+5 / 2}\right),
$$

we thus obtain the following composite profile for the inner three layers:

$$
\ell_{22}^{+ \text {Inner }}=c_{22}^{\prime} y^{+2}\left(1+\left(\frac{y^{+}}{y_{\text {sub }}^{+}}\right)^{4}\right)^{1 / 8}\left(1+\left(\frac{y^{+}}{y_{\text {buf }}^{+}}\right)^{4}\right)^{-3 / 8} .
$$

Similarly, for the outer flow, we have the composite profile connecting the bulk layer and core layer together:

$$
\ell_{22}^{+ \text {Outer }}=\frac{\kappa_{22} R e_{\tau}}{4 Z_{c}^{\prime}}\left(1-r^{4}\right)\left(1+\left(\frac{r_{\text {core }}}{r}\right)^{2}\right)^{1 / 2},
$$


where $Z_{c}^{\prime}=\sqrt{1+r_{\text {core }}^{2}}$. Applying the multiplicative rule (A 5) again for (A 7) and (A 8), with the common state $\ell_{22}^{+C o m m o n}=\kappa_{22} y^{+}=\kappa_{22} R e_{\tau}(1-r)$, we thus obtain $(2.31)$

$$
\begin{aligned}
\ell_{22}^{+} & =\ell_{22}^{+ \text {Inner }} \ell_{22}^{+ \text {Outer }} / \ell_{22}^{+ \text {Common }} \\
& =c_{22} y^{+2}\left(1+\left(\frac{y^{+}}{y_{\text {sub }}^{+}}\right)^{4}\right)^{1 / 8}\left(1+\left(\frac{y^{+}}{y_{\text {buf }}^{+}}\right)^{4}\right)^{-3 / 8} \frac{1-r^{4}}{4(1-r)}\left(1+\left(\frac{r_{\text {core }}}{r}\right)^{2}\right)^{1 / 2} .
\end{aligned}
$$

Note that $c_{22}=c_{22}^{\prime} / Z_{c}^{\prime}$ has been substituted in (A 9). Therefore, by the same procedure developed in Part 1, we obtain the four-layer formula for $\ell_{22}$. This procedure can be similarly applied for $\ell_{11}$ and $\ell_{33}$ to yield (2.32) and (2.37).

\section{REFERENCES}

Adrian, R. J. 2007 Hairpin vortex organization in wall turbulence. Phys. Fluids 19, 041301.

AfZAL, N. 1982 Fully developed turbulent flow in a pipe: an intermediate layer. Ing.-Arch. 52, 355-377.

Ahn, J. S., LeE, J. H., Lee, J., KAnG, J.-H. \& Sung, H. J. 2015 Direct numerical simulation of a $30 R$ long turbulent pipe flow at $R e_{\tau}=3008$. Phys. Fluids 27, 065110.

Ahn, J., LeE, J. \& Sung, H. J. 2017 Contribution of large-scale motions to the Reynolds shear stress in turbulent pipe flows. Intl J. Heat Fluid Flow 66, 209-216.

Alamo, J. C., Jimenez, J., Zandonade, P. \& Moser, R. 2006 Self-similar vortex clusters in the turbulent logarithmic region. J. Fluid Mech. 561, 329-358.

Alfredsson, P. H., Orlu, R. \& Segalini, A. 2012 A new formulation for the streamwise turbulence intensity distribution in wall-bounded turbulent flows. Eur. J. Mech. (B/Fluids) 36, $167-175$.

Alfredsson, P. H., Segalini, A.\& ORlu, R. 2011 A new scaling for the streamwise turbulence intensity in wall-bounded turbulent flows and what it tells us about the outer peak. Phys. Fluids 23, 041702.

Barenblatt, G. I. 1993 Scaling laws for fully developed turbulent shear flows. Part 1. Basic hypotheses and analysis. J. Fluid Mech. 248, 513-520.

Barenblatt, G. I. \& Chorin, A. J. 2004 A mathematical model for the scaling of turbulence. Proc. Natl Acad. Sci. USA 101 (42), 15023-15026.

Bradshaw, P. 1977 Compressible turbulent shear layers. Annu. Rev. Fluid Mech. 9, 33-52.

Buschmann, M. H., Indinger, T. \& GadelhaK, M. 2009 Near-wall behavior of wall-bounded flows. Intl J. Heat Fluid Flow 30, 993-1006.

Cantwell, B. J. 2002 Introduction to Symmetry Analysis. Cambridge University Press.

Chen, X. \& Hussain, F. 2017 Similarity transformation for equilibrium boundary layers, including effects of blowing and suction. Phys. Rev. Fluids 2, 034605.

Chen, X., Hussain, F. \& She, Z. S. $2016 a$ Predictions of canonical wall-bounded turbulent flows via a modified $k-\omega$ equation. J. Turbul. 18 (1), 1-35.

Chen, X., Hussain, F. \& SHE, Z. S. $2016 b$ Bulk flow scaling for turbulent channel and pipe flows. Europhys. Lett. 115, 34001.

CHEn, X.\& SHE, Z. S. 2016 Analytic prediction for planar turbulent boundary layers. Science China Phycis, Mech. Astron. 59 (11), 114711.

Chen, X., Wei, B. B., Hussain, F. \& She, Z. S. 2015 Anomalous dissipation and kinetic-energy distribution in pipes at very high Reynolds numbers. Phys. Rev. E 93, 011102(R).

Davidson, P. A., Kaneda, Y., Moffatt, K. \& SReenivasan, K. R. 2011 A Voyage Through Turbulence. Cambridge University Press.

Davidson, P. A.\& Krogstad, P.-A. 2009 A simple model for the streamwise fluctuations in the log-law region of a boundary layer. Phys. Fluids 21, 055105. 
DegraAfF, D. B.\& Eaton, J. K. 2000 Reynolds-number scaling of the flat plate turbulent boundary layer. J. Fluid Mech. 422, 319-346.

Del Alamo, J. C. \& Jimenez, J. 2006 Linear energy amplification in turbulent channels. J. Fluid Mech. 559, 205-213.

Frewer, M. \& Khujadze, G. 2016 Comments on Janocha et al. Lie symmetry analysis of the Hopf functional-differential equation. Symmetry 2015, 7, 1536c1566. Symmetry 8 (23).

Hoyas, S. \& Jimenez, J. 2006 Scaling of the velocity fluctuations in turbulent channels up to $R e_{\tau}=2003$. Phys. Fluids 18 (1).

Hultmark, M. 2012 A theory for the streamwise turbulent fluctuations in high Reynolds number pipe flow. J. Fluid Mech. 707, 575-584.

Hultmark, M., Vallikivi, M., Bailey, S. C. C. \& Smits, A. J. 2012 Turbulent pipe flow at extreme Reynolds numbers. Phys. Rev. Lett. 108, 094501.

Hultmark, M., Vallikivi, M., Bailey, S. C. C. \& Smits, A. J. 2013 Logarithmic scaling of turbulence in smooth- and rough-wall pipe flow. J. Fluid Mech. 728, 376-395.

Hutchins, N. \& MARUSiC, I. $2007 a$ Evidence of very long meandering streamwise strucutres in the logarithmic region of turbulent boundary laters. J. Fluid Mech. 579, 1-28.

Hutchins, N. \& Marusic, I. $2007 b$ Large-scale influences in near-wall turbulence. Phil. Trans. R. Soc. Lond. A 365, 647-664.

Hutchins, N., Nickels, T. B., Marusic, I. \& Chong, M. S. 2009 Hot-wire spatial resolution issues in wall-bounded turbulence. J. Fluid Mech. 635, 103-136.

Iwamoto, K., Suzuki, Y. \& KASAGi, N. 2002 Database of fully developed channel flow. Tech. Rep. ILR-0201, see http://www.thtlab.t.utokyo.ac.jp.

Jimenez, H. 2012 Cascades in wall-bounded turbulence. Annu. Rev. Fluid Mech. 44, 27-45.

Jones, M. B., Nickels, T. B. \& MARUSiC, I. 2008 On the asymptotic similarity of the zeropressure-gradient turbulent boundary layer. J. Fluid Mech. 616, 195-203.

Kim, J., Moin, P. \& Moser, R. 1987 Turbulence statistics in fully developed channel flow at low Reynolds number. J. Fluid Mech. 177, 133-166.

KLEWICKI, J. C. 2013 Self-similar mean dynamics in turbulent wall flows. J. Fluid Mech. 718, $596-621$.

Klewicki, J., Chin, C., Blackburn, H., Ooi, A. \& Marusic, I. 2012 Emergence of the four layer dynamical regime in turbulent pipe flow. Phys. Fluids 24, 045107.

KLEWICKI, J. C. \& FALCO, R. E. 1990 On accurately measuring statistics associated with small-scale structure in turbulent boundary layers using hot-wire probes. J. Fluid Mech. 219, 119-142.

Laval, J.-P., Vassilicos, J. C., Foucaut, J.-M. \& Stanislas, M. 2017 Comparison of turbulence profiles in high-Reynolds-number turbulent boundary layers and validation of a predictive model. J. Fluid Mech. 814, R2.

Lee, J. \& Moser, I. 2015 Direct numerical similation on channel flow up to $R e_{\tau} \approx 5200$. J. Fluid Mech. 774, 395-415.

Lozano-Duran, A. \& Jimenez, J. 2014 Effect of the computational domain on direct simulations of turbulent channels up to $R e_{\tau}=4200$. Phys. Fluids 26, 011702.

Luchini, P. 2017 Universality of the turbulent velocity profile. Phys. Rev. Lett. 118, 224501.

L'vov, V. S., Procaccia, I. \& Rudenko, O. 2008 Universal model of finite Reynolds number turbulent flow in channels and pipes. Phys. Rev. Lett. 100 (5), 054504.

Marati, N., Davoudi, J., Casciola, C. M. \& Eckhardt, B. 2006 Mean profiles for a passive scalar in wall-bounded flows from symmetry analysis. J. Turbul. 7, N61.

MARUsic, I., BAARS, W. J. \& Hutchins, N. 2017 Scaling of the streamwise turbulence intensity in the context of inner-outer interactions in wall turbulence. Phys. Rev. Fluids 2, 100502.

Marusic, I., Chauhan, K. A., Kulandaivelu, V. \& Hutchins, N. 2015 Evolution of zero-pressure-gradient boundary layers from different tripping conditions. J. Fluid Mech. 783, $379-411$.

MARUsic, I. \& KUnKel, G. J. 2003 Streamwise turbulent intensity formulation for flat plate boundary layers. Phys. Fluids 15, 24-61. 
Marusic, I., Mathis, R. \& Hutchins, N. $2010 a$ Predictive model for wall-bounded turbulent flow. Science 329 (5988), 193-196.

Marusic, I., McKeon, B. J., Monkewitz, P. A., Nagib, H. M., Smits, A. J. \& Sreenivasan, K. R. $2010 b$ Wall-bounded turbulent flows at high Reynolds numbers: recent advances and key issues. Phys. Fluids 22 (6), 065103.

Mathis, R., Hutchins, N.\& Marusic, I. 2009 Large-scale amplitude modulation of the small-scale structures in turbulent boundary layers. J. Fluid Mech. 628, 311-337.

McKeon, B. J. 2017 The engine behind (wall) turbulence: perspectives on scale interactions. J. Fluid Mech. 817, P1.

McKeon, B. J. \& Sharma, A. S. 2010 A critical-layer framework for turbulent pipe flow. J. Fluid Mech. 658, 336-382.

Meneveau, C. \& Marusic, I. 2013 Generalized logarithmic law for high-order moments in turbulent boundary layers. J. Fluid Mech. 719, R1.

Metzger, M. M., Klewicki, J., Bradshaw, K. L. \& SAdr, R. 2001 Scaling the near-wall axial turbulent stress in the zero pressure gradient boundary layer. Phys. Fluids 13, 1819.

Motrref, R., Sharma, A. S., Tropp, J. A. \& McKeon, B. J. 2013 Model-based scaling of the streamwise energy density in high-Reynolds number turbulent channels. J. Fluid Mech 734, 275-316.

Monkewitz, P. A., Chauhan, K. A. \& Nagib, H. M. 2007 Self-consistent high-Reynolds-number asymptotics for zero-pressure-gradient turbulent boundary layers. Phys. Fluids 19 (11).

Morkovin, M. V. 1962 Effects of compressibility on turbulent flows. In Mecanique de la Turbulence (ed. A. Favre), pp. 367-380. Centre National de la Recherche Scientifique (CNRS).

Morrison, J. F., McKeon, B. J., Jiang, W. \& Smits, A. J. 2004 Scaling of the streamwise velocity component in turbulent pipe flow. J. Fluid Mech. 508, 99-131.

NaGiB, H. M. \& Chauhan, K. A. 2008 Variations of von Kármán coefficient in canonical flows. Phys. Fluids 20 (10).

Nagib, H. M., Monkewitz, P. A., Mascotelli, L., Fiorini, T., Bellani, G., Zheng, X. \& Talamelli, A. 2017 Centerline Kármán 'constant' revisited and contrasted to log-layer Kármán constant at CICLoPE. In 10th International Symposium on Turbulence and Shear Flow Phenomena (TSFP10), Chicago, USA.

OBERLACK, M. 2001 A unified approach for symmetries in plane parallel turbulent shear flows. J. Fluid Mech. 427, 299-328.

Oberlack, M. \& Rosteck, A. 2010 New statistical symmetries of the multi-point equations and its importance for turbulent scaling laws. Discrete Contin. Dyn. Syst. S 3, 451-471.

ÖRlü, R.\& Alfredsson, P. H. 2013 Comment on the scaling of the near-wall streamwise variance peak in turbulent pipe flows. Exp. Fluids 54, 1431.

Panton, R. L. 2007 Composite asymptotic expansions and scaling wall turbulence. Phil. Trans. R. Soc. Lond. A 365, 733-754.

Perry, A. E. \& Chong, M. S. 1982 On the mechanism of wall turbulence. J. Fluid Mech. 119.

Perry, A. E., Henbest, S. M. \& ChOng, M. S. 1986 A theoretical and experimental study of wall turbulence. J. Fluid Mech. 165, 163-199.

Pope, S. B. 2000 Turbulent Flows. Cambridge University Press.

Rosenberg, B. J., Hultmark, M., Vallikivi, M., Bailey, S. C. C. \& Smits, A. J. 2013 Turbulence spectra in smooth- and rough-wall pipe flow at extreme Reynolds numbers. J. Fluid Mech. 731, 46-63.

She, Z. S., Chen, X. \& Hussain, F. 2017 Quantifying wall turbulence via a symmetry approach. Part 1. A Lie group theory. J. Fluid Mech. 827, 322-356.

She, Z. S., Chen, X., Wu, Y. \& Hussain, F. 2010 New perspective in statistical modeling of wall-bounded turbulence. Acta Mechanica Sin. 26 (6), 847-861.

She, Z. S. \& Leveque, E. 1994 Universal scaling laws in fully developed turbulence. Phys. Rev. Lett. 72 (3), 336.

She, Z. S., Wu, Y., Chen, X. \& Hussain, F. 2012 A multi-state description of roughness effects in turbulent pipe flow. New J. Phys. 14 (9), 093054. 
Sillero, J. A., Jimenez, J. \& Moser, R. 2013 One-point statistics for turbulent wall-bounded flows at Reynolds numbers up to $\delta^{+}=2000$. Phys. Fluids 25, 105102.

SMits, A. J. 2010 High Reynolds number wall-bounded turbulence and a proposal for a new eddy-based model. In Turbulence and Interations (ed. M. Deville, T. H. Le \& P. Sagaut), pp. 51-62. Springer.

Smits, A. J. \& Marusic, I. 2013 Wall-bounded turbulence. Phys. Today 66 (9), 25.

SReEnivasan, K. R. 1988 Turbulence Management and Relaminarization. Springer.

SReEnivasan, K. R. \& SAHAY, A. 1997 The persistence of viscous effects in the overlap region, and the mean velocity in turbulent pipe and channel flows. In Self-Sustaining Mechanisms of Wall Turbulence (ed. R. Panton), pp. 253-272. Comp. Mech. Publ.

Townsend, A. A. 1976 The Structure of Turbulent Shear Flow. Cambridge University Press.

Vallikivi, M., Ganapathisubramani, B. \& Smits, A. J. 2015 Spectral scaling in boundary layers and pipes at very high Reynolds numbers. J. Fluid Mech. 771, 303-326.

VAN DYKe, M. 1964 Perturbation Methods in Fluid Mechanics, vol. 964. Academic.

Vassilicos, J. C. 2015 Dissipation in turbulent flows. Annu. Rev. Fluid Mech. 47 (1), 95-114.

Vassilicos, J. C., Laval, J.-P., Foucaut, J.-M. \& Stanislas, M. 2015 The streamwise turbulence intensity in the intermediate layer of turbulent pipe flow. J. Fluid Mech. 774, 324-341.

Vincenti, P., Klewicki, J., Morrill-Winter, C., White, C. M. \& Wosnik, M. 2013 Streamwise velocity statistics in turbulent boundary layers that spatially develop to high Reynolds number. Exp. Fluids 54, 1629.

Wei, T., Fife, P., Klewicki, J. \& Mcmurtry, P. 2005 Properties of the mean momentum balance in turbulent boundary layer, pipe and channel flows. J. Fluid Mech. 522, 303-327.

WilcoX, D. C. 2006 Turbulence Modeling for CFD. DCW Industries La Canada.

Willert, C. E., Soria, J., Stanislas, M., Klinner, J., Amili, O., Eisfelder, M., Cuvier, C., Bellani, G., Fiorini, T. \& Talamelli, A. 2017 Near-wall statistics of a turbulent pipe flow at shear Reynolds numbers up to 40000 . J. Fluid Mech. 826, R5.

Wosnik, M., Castillo, L. \& George, W. K. 2000 A theory for turbulent pipe and channel flows. J. Fluid Mech. 421, 115-145.

Wu, B., BI, W. T., Hussain, F. \& She, Z. S. 2017 On the invariant mean velocity profile for compressible turbulent boundary layers. J. Turbul. 18 (2), 186-202.

WU, X. H. \& MoIN, P. 2008 Direct numerical simulation on the mean velocity characteristics in turbulent pipe flow. J. Fluid Mech. 608, 5-41.

Wu, Y., Chen, X., She, Z. S. \& Hussain, F. 2013 On the Kármán constant in turbulent channel flow. Phys. Scr. 2013 (T155), 014009.

Zhang, Y. S., Bi, W. T., Hussain, F., Li, X. L. \& She, Z. S. 2012 Mach-number-invariant mean-velocity profile of compressible turbulent boundary layers. Phys. Rev. Lett. 109, 054502. 